\title{
Underpredicting Pain: An experimental investigation into the benefits and risks
}

\author{
Kaya J. Peerdeman ${ }^{a, b,{ }^{*}}$, Andrew L. Geers ${ }^{c}$, Delia Della Porta ${ }^{a}$, Dieuwke S. Veldhuijzen ${ }^{\text {a,b }}$, Irving Kirsch ${ }^{d}$ \\ a Unit Health, Medical and Neuropsychology, Leiden University, Leiden, the Netherlands \\ ${ }^{\mathrm{b}}$ Leiden Institute for Brain and Cognition, Leiden University, Leiden, the Netherlands \\ c University of Toledo, Toledo, OH, USA \\ d Program in Placebo Studies, Harvard Medical School, Boston, MA, USA
}

* Corresponding author: K. J. Peerdeman.

Address: Unit Health, Medical and Neuropsychology, Leiden University, P.O. Box 9555, 2300 RB Leiden, the Netherlands. Tel.: +31-71-527-3622. Fax: +31-71-527-3619.

E-mail address: k.j.peerdeman@fsw.leidenuniv.nl

https://www.universiteitleiden.nl/en/staffmembers/kaya-peerdeman\#tab-1

Note: The current version of the article is a preprint and has not yet been subject to peer review. It is not the final published version.

\begin{abstract}
Expectancies can shape pain and other experiences. Generally, experiences change in the direction of what is expected (i.e., assimilation effects), as seen with placebo effects. However, in case of large expectation-experience discrepancies, experiences might change away from what is expected (i.e., contrast effects). Previous research has demonstrated contrast effects on various outcomes, but not pain. We investigated the effects of strong underpredictions of pain on experienced pain intensity. Additionally, we assessed related outcomes including (certainty of) expectations, fear of pain, pain unpleasantness, autonomic responses, and trust. Healthy participants (Study 1: $n=81$, Study 2: $n=123$ ) received verbal suggestions that subsequent heat stimuli would be moderately or highly painful (correct prediction), mildly painful (medium underprediction; Study 2 only), or non-painful (strong underprediction). Both studies showed that participants experienced less intense pain upon strong underprediction than upon correct prediction (i.e., assimilation). Expected pain, fear of pain, and pain unpleasantness were generally also lowered. However, strong underprediction simultaneously lowered certainty of expectations and trust in the experimenter. Study 2 indicated that the effects of strong underprediction versus medium underprediction generally did not differ. Moreover, Study 2 provided some indications for reduced heart rate and skin conductance levels, but increased skin conductance responses upon strong underprediction. In conclusion, even strong underpredictions of pain can reduce pain (i.e., cause assimilation), although not significantly more than medium underpredictions. However, strong underpredictions can cause uncertainty and undermine trust. These findings suggest that healthcare providers may wish to be cautious with providing overly positive information about painful medical procedures.
\end{abstract}

Keywords: expectancies; verbal suggestion; assimilation; contrast effect; pain; trust; placebo effect 


\section{Introduction}

Expectancies have repeatedly been found to influence pain experiences and are considered to be a core mechanism of placebo and nocebo effects [5,14,25,31,32]. In line with response expectancy theory [31,32], predictive coding models [10], and other theoretical accounts [8,40,57], pain experiences are generally found to change in the direction of what one expects, referred to as assimilation effects [43-45,54]. As such, shaping positive expectancies may improve pain treatment [16]. Under certain circumstances, however, expectancies might have counteractive effects. Most notably, the affective expectation model [57], as other models $[8,40,41]$, suggests that experiences can change away from expectations, referred to as contrast effects. Contrast effects have been observed in various research fields, particularly when there is a large discrepancy between expectations and experiences $[1,7,11,18,19,33,40,41]$. However, to our knowledge, currently no empirical evidence exists for contrast effects on pain. This may be partly due to the use of relatively small and hence unnoticed expectation-experience discrepancies in previous research.

Alternatively, the ambiguous nature of pain experiences may inherently favor assimilation, even when expectation violations are large, but there might be limits to this. In line with earlier research [33], recent experiments demonstrated a tipping point at which large discrepancies between predicted and actual pain intensity led to less assimilation than moderate discrepancies [24]. Moreover, there are some indications that even when underprediction of pain leads to assimilation, negative effects in the form of increased autonomic responses, uncertainty, and disruptions on an attention task can occur [2,3]. Observational and qualitative research further indicates that patients commonly hold overly positive expectations of medical treatments, and violation of these expectations has been related to disappointment, distress, and treatment dissatisfaction $[13,23,27,35]$. Expectation violations might also undermine trust in the caregiver, which is core in health care [22] and in generating placebo effects [16]. Further research is required to obtain a more comprehensive understanding of the benefits and risks of large expectationexperience discrepancies on pain, particularly when expectancies are overly positive. This will provide essential insights into how painful medical procedures and analgesic treatments can best be introduced to patients in clinical practice.

In two preregistered experiments, we investigated the effects of strong underpredictions of pain. Our primary hypothesis was that a suggestion of no pain from a moderately or highly painful heat stimulus (strong underprediction) would increase experienced pain intensity (i.e., elicit a contrast effect) as compared to a correct prediction. In Study 2, we also compared this to a medium underprediction of pain to assess possibly reduced assimilation upon large versus medium expectation-experience discrepancies. Secondary, we hypothesized that effects on pain expectations upon undergoing the expectation-experience (mis)match would be comparable to effects on pain intensity. We additionally explored effects on expected and experienced pain during multiple trials, including upon repetition of the suggestions, as well as effects on expectation certainty, fear of pain, pain unpleasantness, heart rate, and skin conductance. Last, we explored additional psychological outcomes (e.g., trust, discrepancy awareness) and possible moderation by individual characteristics (e.g., optimism).

\section{Study 1}

The primary aim of Study 1 was to assess if strong underprediction of pain could increase experienced pain intensity, i.e., evoke a contrast effect. Participants were randomly assigned to one 
of the two experimental conditions that differed only in the verbal suggestions provided: 1) Strong underprediction condition, in which suggestions that 2 series of 4 presentations of a heat stimulus would all be non-painful were given, and 2) Correct prediction condition, with suggestions that the stimulus would be moderately painful. The primary outcome was pain intensity experienced during the first post-suggestion trial given after the condition-specific suggestion to examine initial responses. The secondary outcome was expected pain intensity upon undergoing the expectationexperience (mis)match. All additional outcomes were exploratory.

\section{Methods Study 1}

\section{Participants}

In Study 1, 82 healthy adults were included. This sample size was deemed sufficient to observe a small to medium effects size with the planned primary analyses, i.e., an analysis of covariance analyzing the effect of the verbal suggestions on experienced pain intensity during the first post-suggestion trial, controlled for average experienced pain intensity during the 3 high reference trials. First, the required sample size for an independent $\mathrm{t}$-test was determined in $\mathrm{G}$ *power (two-sided, $d=.35, \alpha=.05$, power $=.80$ ), which was then corrected to account for a covariate that correlates strongly with the outcome ( $r=.85$, [42]) with Borm and Fransen's formula [9]. An error margin of $10 \%$ was added, resulting in a target total sample size of 82 . Eligibility criteria were: age 1830 years, good understanding of written and spoken English, no severe physical or psychological morbidity (e.g., heart and lung diseases, or DSM-V psychiatric disorders) that could adversely affect study participation, no chronic ( $\geq 6$ months) past or present pain complaints, no or low current pain ( $\leq 3$ on 0-10 numerical rating scale; NRS), no current use of any medication, no pacemaker, and no pregnancy. In addition, participants were instructed to not use any medication, alcohol, or other drugs in the 24 hours prior to the lab session.

\section{Procedure}

Data collection took place in a standard psychophysiology lab at the Faculty of Social and Behavioural Sciences, Leiden University, Leiden, the Netherlands from March to September 2017. The study protocol was approved by Leiden University's Psychology Research Ethics Committee (CEP17-0314_152) and preregistered at the Netherlands Trial Register (NL6228).

Participants were recruited via the university's online participant recruitment system, social media, flyers around the university, and personal communication. Potential participants were informed that the purpose of the study was to investigate pain sensitivity during heat stimuli. Upon registering, participants filled in a screening questionnaire and additional questionnaires (assessing demographics, optimism, general trust, pain catastrophizing, and socially desirable responding) via an online system (Qualtrics, Provo, UT, USA; approx. $10 \mathrm{~min}$ ) for which they separately provided digital informed consent.

Eligible participants were invited to make an appointment for the lab session. To maximize standardization and blinding, two female experimenters (trained bachelor and master psychology students) carried out the entire procedure according to a detailed protocol. Experimenter A, who supervised the outcome assessments, remained blinded throughout the whole procedure; Experimenter $B$ was blinded until she gave the condition-specific verbal suggestion, during which Experimenter $A$ briefly left the lab with an excuse. Participants were not informed about the different experimental conditions until debriefing. 
Once in the lab, after an explanation of the procedures and a brief check of the eligibility criteria, participants provided written informed consent. Subsequently, electrodes for the autonomic measurements were attached, upon which participants filled out computerized questionnaires (assessing trait and state anxiety and fatigue). Five to 10 minutes after electrode attachment, a 3minute resting measurement of heart rate and skin conductance was taken. Skin conductance and heart rate were recorded continuously with epochs of interest marked.

Subsequently, warmth and pain perception thresholds were determined to familiarize participants with the heat stimuli. Next, a step-up heat pain calibration procedure was used to determine the temperature at which participants experienced moderately high pain. Participants received 3 presentations of a non-painful heat stimulus prior to the step-up procedure, and 3 presentations of a moderately painful heat stimulus afterwards, which served as reference points for the test.

In the subsequent manipulation and test phase, participants received either a suggestion of no pain (Strong underprediction condition) or a suggestion of moderately high pain (Correct prediction condition) depending on the condition to which they were randomized (using a randomization sequence generated by an independent researcher with an online random number generator, stratified by sex, 1:1 ratio, blocks of 4 and 6 and concealed by using sequentially numbered, opaque, sealed envelopes). All participants then received the moderately painful heat stimulus 4 times (after the thermode had been placed on a novel spot on the arm to avoid sensitization). Immediately after, the condition-specific verbal suggestion was repeated and 4 additional trials were given. Prior to each trial, participants rated expected pain intensity, certainty of the expectation, and fear of the upcoming stimulus. Directly after each trial, participants rated experienced pain intensity and pain unpleasantness.

The experimental session was concluded with a second assessment of state anxiety and several exit questions were filled in digitally, followed by an oral debriefing. All participants were reimbursed for their participation. The total duration of the test session was around 1.15 hours. See Figure 1 for an overview of the procedure.

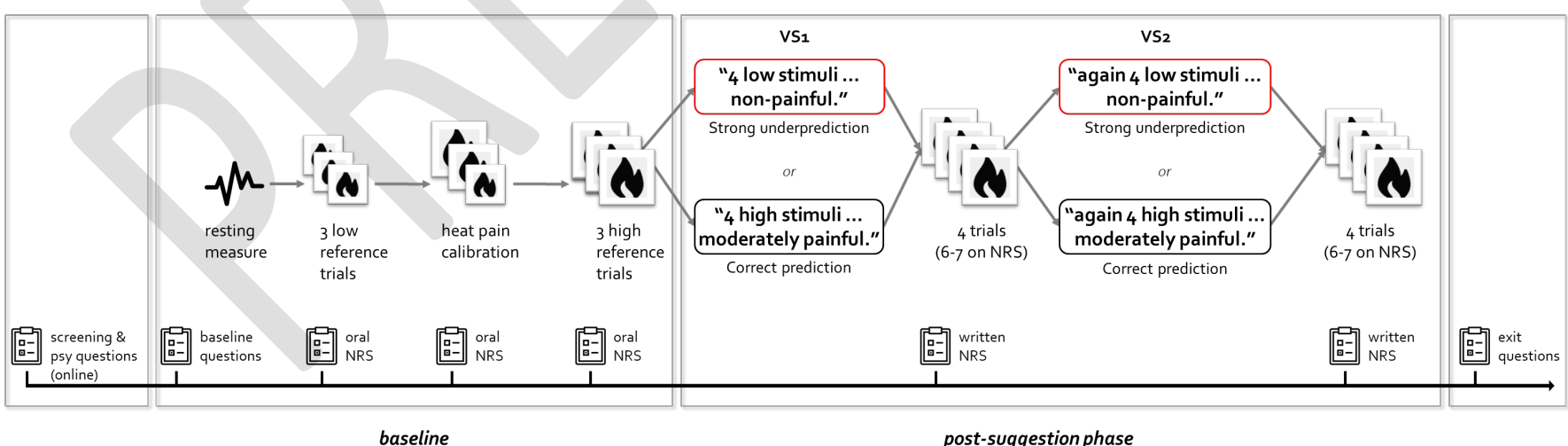

$\boldsymbol{N}=$ thermal pain stimuli $\quad\left[\begin{array}{ll}0 \\ -1\end{array}\right]=$ assessments of pain, etc.

Figure 1.

Overview procedure with all measures taken in Study 1.

NRS = numerical rating scale; VS = verbal suggestion 


\section{Pain evocation and assessment}

Warmth and painful sensations were evoked by applying heat stimuli to the volar surface of the non-dominant forearm with a TSA-II NeuroSensory Analyzer (Medoc, Ramat Yishai, Israel) using a standard 30x30mm Advanced Thermal Stimulation (ATS) thermode and accompanying Medoc Main Station software (version 6.3.6.19.9).

Warmth and pain thresholds. Following a practice trial, the warmth detection threshold was determined by administering 3 heat stimuli of ascending intensity until the participant indicated by a mouse click feeling warmth for the first time $\left(1^{\circ} \mathrm{C} / \mathrm{sec}\right.$ ramp up, $8^{\circ} \mathrm{C} / \mathrm{sec}$ ramp down from/to a baseline of $32^{\circ} \mathrm{C}$ ). The inter stimulus interval (ISI) was set at 10 seconds. The average of these 3 measurements was taken as the threshold in line with validated procedures [48]. The same procedure was used for the heat pain threshold detection when participants indicated feeling pain for the first time.

Heat pain calibration step-up procedure and reference stimuli. First, 3 presentations of a warm, non-painful stimulus were given to serve as a reference for the strong underprediction suggestion $\left(35^{\circ} \mathrm{C}\right.$; low reference stimulus). Subsequently, we used a step-up procedure in which stimuli of increasing temperatures were given (starting at $39^{\circ} \mathrm{C}$, steps of $2^{\circ} \mathrm{C}$ until $43^{\circ} \mathrm{C}$, then steps of $.5^{\circ} \mathrm{C}$ until max $49.5^{\circ} \mathrm{C}$ ) until the participants reported moderately high pain. Moderately high pain was defined as a pain intensity rating between 6 and 7 on an NRS ranging from 0 (no pain at all) to 10 (worst pain imaginable). Another 3 trials of this moderately high pain intensity were given to serve as a reference for the post-suggestion trials ( $\mathrm{M}=47.4^{\circ} \mathrm{C}, \mathrm{SD}=1.9^{\circ} \mathrm{C}$, high reference stimulus). To increase focus on the low and high reference stimuli, and thereby increase chances that participants became aware of the (mis)match, participants were asked whether the trials felt approximately the same. All stimuli were set to have a duration of approximately 8 seconds $\left(4 \mathrm{sec}\right.$ at peak, $8^{\circ} \mathrm{C} / \mathrm{sec}$ ramp up and down from/to $32^{\circ} \mathrm{C}$ baseline) and were separated by an ISI of 10 seconds. Due to Medoc software issues, stimuli were actually at peak for 4.0 to 10.6 seconds ( $M=8.2, S D=1.1 \mathrm{sec}$ ). For autonomic analyses, post hoc corrections of epoch duration were done by hand to cover actual peak duration.

Post-suggestion trials. Participants received two series of 4 trials of the same moderately high intensity $\left(\mathrm{M}=47.4^{\circ} \mathrm{C}, \mathrm{SD}=1.9^{\circ} \mathrm{C}\right)$ and of the same duration as the high reference stimulus, with an ISI of 30 seconds.

Pain ratings. Participants rated experienced pain intensity directly after each trial on an NRS ranging from 0 (no pain at all) to 10 (worst pain imaginable) with the option to answer with 1 decimal precision. For the post-suggestion trials, participants additionally rated for each trial: 1) expected pain intensity, 2) certainty of pain expectation, 3) fear of upcoming pain stimulus, and 4) pain unpleasantness on 0-10 NRSs. The distinction between pain intensity and unpleasantness was explained according to previously established and frequently used instructions [47]. Participants provided their ratings verbally for the threshold, calibration, and reference stimuli. For the postsuggestion trials, participants wrote the ratings down, to reduce possible response biases.

\section{Verbal suggestion regarding pain intensity}

In the Strong underprediction condition, the first 4 post-suggestion trials were suggested to be non-painful by the experimenter using the following suggestion "You'll now get 4 low stimuli. These are the same as the low stimuli you felt before, at the beginning of the previous test. Based on the previous test, I expect that you will experience them as non-painful. But please rate them as you feel them now." 
In the Correct prediction condition, participants were correctly informed that the trials would be moderately painful using the following suggestion: "You'll now get 4 high stimuli. These are the same as the high stimuli you felt before, at the end of the previous test. Based on the previous test, I expect that you will experience them as moderately painful. But please rate them as you feel them now."

The condition-specific suggestion was repeated before giving the 4 additional trials: "You will now again get 4 low stimuli, that should be non-painful." (Strong underprediction condition), or "You will now again get 4 high stimuli, that should be moderately painful." (Correct prediction condition). To enhance the chances that participants would notice the (mis)match between the instruction and the sensation, all participants were instructed to attend closely to their sensations during the tests and to compare those sensations to the reference stimuli.

\section{Autonomic measures}

Electrocardiography (ECG) and skin conductance were measured with an MP150 system, including an ECG100C amplifier (1000 Hz, gain 1000, $35 \mathrm{~Hz}$ low pass filter, $1 \mathrm{~Hz}$ high pass filter), a GSR100C amplifier (1000 Hz, gain $5 \mu \mathrm{mho} / \mathrm{V}, 1 \mathrm{~Hz}$ low pass filter), and STP100C and UIM100C modules, with AcqKnowledge software (version 5.0) (BIOPAC Systems Inc., Goleta, CA, USA). For ECG recordings, disposable electrodes (Kendall 200 Foam Electrode, Covidien, Mansfield, MA, USA) were placed on the sternum and the left lower rib, after abrading the skin. For skin conductance recordings, disposable $\mathrm{Ag} / \mathrm{AgCl}$ electrodes (EL507-10, BIOPAC Systems Inc., Goleta, CA, USA) were placed on the medial phalanges of the index and middle finger of the non-dominant hand, after the skin was cleaned with water and dried. Epochs were marked from peak temperature onset to offset by manually sending triggers via E-prime 2.0 software (Psychology Software Tools, Inc., Sharpsburg, PA, USA). For skin conductance level and response measures, a latency window of 1 second after peak temperature onset until 4 seconds after peak temperature offset was used [12]. In addition, based on visual inspection and taking rise time into account, post hoc sensitivity analyses were run for skin conductance response with an extended latency window of 0 seconds after temperature rise onset until 4 seconds after peak offset. Inspection of the ECG and skin conductance data, calculation of the average heart rate (bpm) and skin conductance levels $(\mu \mathrm{S})$, and detection of skin conductance response $(\mu \mathrm{S})$ during rest, reference stimuli, and post-suggestion trials was done with PhysioData Toolbox (version 0.5.0) [51]. Skin conductance response amplitudes during these epochs were determined using an adjusted version of EDA toolbox [28] in Matlab (R2018b).

\section{Psychological questionnaires}

In addition to basic assessments of demographic characteristics, participants completed several questionnaires to assess possible moderators of the effects: 1 ) optimism (Life Orientation Test - Revised, LOT-R, Cronbach's $\alpha$ in this study $=0.75$ ) [49], 2) general trust (Generalized Trust Scale, GTS, $\alpha=0.82$ ) [58], 3) pain catastrophizing (Pain Catastrophizing Scale, PCS, $\alpha=0.90$ ) [53], 4) ability to modify self-presentation and sensitivity to expressive behaviors of others (subscales of SelfMonitoring Scale - revised, SMS-R, $\alpha=0.78$ and $\alpha=0.70$, respectively) [36], 5) trait anxiety (StateTrait Anxiety Inventory, Trait version, STAI-T, $\alpha=0.89$ ) [52], 6) state anxiety at baseline (State-Trait Anxiety Inventory, short State version, STAI-Ss, $\alpha=0.76$ ) [37], and 7) fatigue at baseline (visual analogue scale from 0 no fatigue at all to 10 worst fatigue imaginable).

Exit questions re-assessed state anxiety (STAI- Ss, $\alpha=0.76$ ) and 0 to 10 visual analogue scales (VAS, with 1 decimal precision) were used to assess participants' previous experience with heat pain 
tests; pain intensity during previous heat pain tests; focus during the tests; confidence in their knowledge of the study purpose; observation of a discrepancy between actual pain intensity versus the low reference, high reference, expected, and instructed pain intensity; trustworthiness of the experimenters; and possible response bias (with 4 items: 1 . concerns about what experimenter thought, 2. change of response to help experimenter, 3 . Sense of owing it to experimenter to report more or less pain, 4. providing answers experimenter wanted to hear). Additional open response questions asked participants to report their view of the purpose and expected results of the study, as well as any further comments they might have. The exit questions are available via https://osf.io/24mu5/ (doi: 10.17605/OSF.IO/24MU5).

\section{Statistical analyses}

To test the primary hypothesis, an analysis of covariance (ANCOVA) was used with condition (Underprediction vs. Correct prediction) as independent variable (IV) and experienced pain intensity during the first post-suggestion trial as dependent variable (DV). Average experienced pain intensity during the 3 high reference trials was included as a covariate to reduce error variance and hence provide more precise estimates of the effects. In addition, we explored participants' pain during the trials using a mixed-model ANCOVA with condition as between-subjects IV, trial as within-subjects IV, experienced pain intensity during each of the 8 trials as DV, and average experienced pain intensity during the 3 high reference trials as covariate.

To test the secondary hypothesis, an analysis of variance (ANOVA) was run with condition as IV and expected pain intensity following the first post-suggestion trial as DV. In addition, an ANOVA with condition as IV and expected pain intensity prior to the first post-suggestion trial as DV was run as a manipulation check. We also explored participants' expectancies regarding all trials with a mixed-model ANOVA.

Certainty of expectations, fear of upcoming pain, and pain unpleasantness during all postsuggestion trials were explored with mixed-model ANOVAs with condition and trial as IVs, and the relevant ratings for all 8 trials as DVs. We similarly explored effects on autonomic responses using ANCOVAs with condition and trial as IVs, and with heart rate, skin conductance level, and skin conductance response during all 8 trials as DVs, controlled for average responses of the relevant measure during the 3 high reference trials.

Effects on psychological outcomes assessed in the exit questionnaire were explored with oneway ANOVAs with condition as IV and the respective measures as DV, with the exception of effects on state anxiety for which an ANCOVA was used with pre-manipulation state anxiety as covariate.

The possible moderating role of individual characteristics was explored by examining the interaction between each individual characteristic and condition in separate multiple regression analyses with experienced pain intensity during the first post-suggestion trial as DV, while controlling for the main effects of the relevant individual measure and condition, as well as average experienced pain intensity during the 3 high reference trials.

All analyses were run in Rstudio (version 1.1.463; R version 3.5.3). Test results were reported with the StatTag plugin [56]. For readability, generally only statistically significant test results ( $\alpha=$ $.05)$ are reported. For $A N(C) O V A s$, generalized eta squared $\left(\eta_{G}{ }^{2}\right)$ was computed, with $0.01,0.06$, and 0.14 signifying small, medium, and large effects, respectively [34]. For repeated measures analyses, a Greenhouse-Geisser correction was used when the sphericity assumption was violated. To evaluate the robustness of the results, the primary analysis (effect of condition on experienced pain intensity during the first post-suggestion trial) was also run: 1 . with the stratification variable sex as an 
additional covariate, 2 . with stimulus peak duration as an additional covariate, and 3 . without any covariates.

\section{Results Study 1}

Data availability

Table SDC-1 presents the descriptives (mean \pm standard deviation) per condition of all reported measures. The data are available via https://osf.io/24mu5/ (doi: 10.17605/OSF.IO/24MU5). [The final analysis scripts and results files will be uploaded upon publication of the paper in a peerreviewed journal.]

\section{Participants}

Of the 82 participants included, one participant dropped out after the first high reference trial (prior to randomization) because they could no longer handle the pain. For analyses, data of the 81 participants who completed participation was available $(50.6 \%$ female; $\mathrm{M}$ age $=22.9, \mathrm{SD}=2.7 ; 50.6 \%$ Dutch nationality; median baseline pain $=0.0$, interquartile range $=0.2$ ).

\section{Effects of verbal suggestions on pain intensity}

Contrary to our primary hypothesis, the strong underprediction of pain led to significantly lower pain intensity than the correct prediction for the first post-suggestion trial, $F(1,78)=19.19, p<$ $0.001, \eta_{G}{ }^{2}=0.20$ (Figures $2 A$ and SDC-1). Sensitivity analyses with the stratification variable sex or stimulus peak duration as an additional covariate and analyses without covariates yielded the same conclusions.

An exploration of pain intensity during all post-suggestion trials indicated a main effect of condition, $F(1,77)=13.48, p<0.001, \eta_{G}{ }^{2}=0.10$, with no significant main effect of trial, nor an interaction effect, demonstrating that the between-group differences persisted over time, also upon a repetition of the verbal suggestion.

A

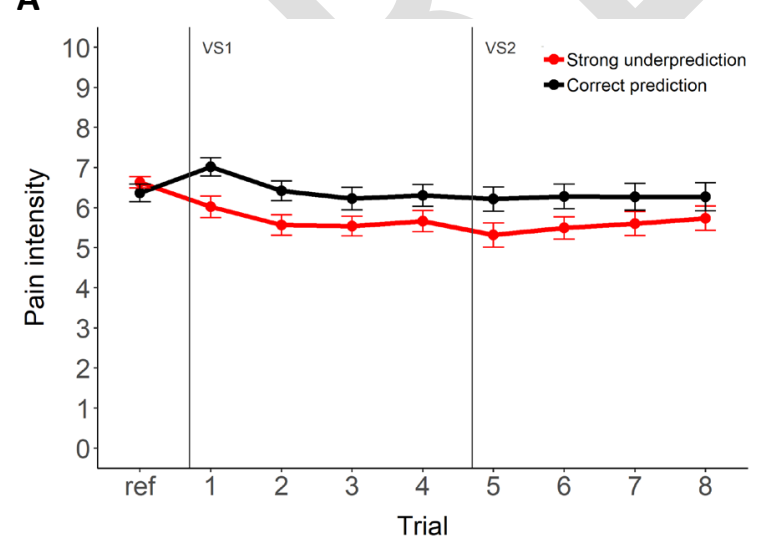

B

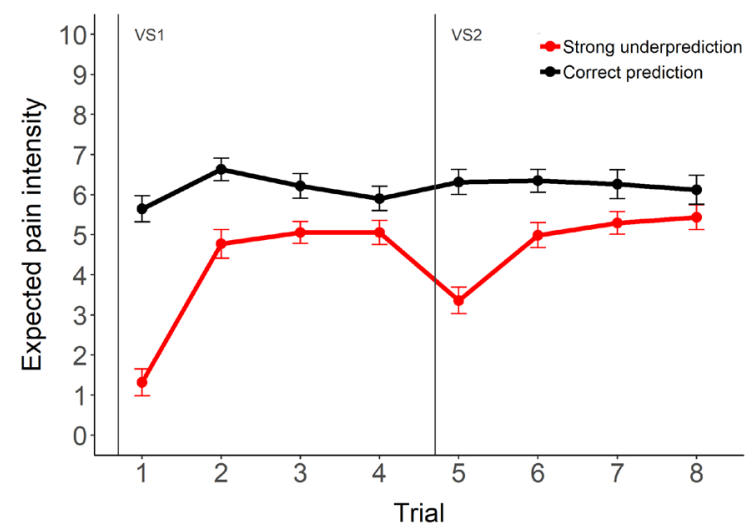

Figure 2.

A. Experienced pain intensity ratings $(\mathrm{M} \pm \mathrm{SE}$ ) upon the 3 high reference trials (average) and the 8 post-suggestion trials, and $B$. Expected pain intensity ratings $(M \pm S E)$ prior to each of the 8 postsuggestion trials, per condition, in Study 1.

ref $=$ average of the 3 high reference trials; VS = verbal suggestion . 


\section{Expected pain intensity}

The strong underprediction led participants to expect significantly less intense pain for the first post-suggestion trial than the correct prediction, $F(1,79)=82.22, p<0.001, \eta_{G}{ }^{2}=0.51$, demonstrating that the verbal suggestions successfully manipulated expectations (Figure 2B). Contrary to our secondary hypothesis, participants in the Strong underprediction condition expected the trial following a violation of their initial expectation of no pain during the first post-suggestion trial to be less painful than participants in the Correct prediction condition, $F(1,78)=15.72, p<0.001$, $\eta_{G}{ }^{2}=0.17$.

An exploration of expected pain intensity during all trials, indicated main effects of condition, $F(1,77)=24.09, p<0.001, \eta_{G}{ }^{2}=0.17$, and trial, $F(4.09,315.24)=31.50, p<0.001, \eta_{G}{ }^{2}=0.12$, and an interaction effect, $F(4.09,315.24)=19.95, p<0.001, \eta_{G}{ }^{2}=0.08$. In the Strong underprediction condition, participants' expectations of pain increased substantially upon experiencing the first postsuggestion trial, decreased upon repetition of the verbal suggestion, and then increased again upon experiencing the subsequent trial, while for participants in the Correct prediction condition their expectations remained stable across trials.

\section{Other pain outcomes}

Certainty of pain expectation. An exploration of certainty of pain expectations for all postsuggestion trials indicated main effects of condition, $F(1,76)=4.08, p=0.047, \eta_{G}{ }^{2}=0.03$, and trial, $F(4.62,351.02)=3.95, p=0.002, \eta_{G}{ }^{2}=0.02$, and an interaction effect, $F(4.62,351.02)=8.06, p<$ $0.001, \eta_{\mathrm{G}}{ }^{2}=0.04$ (Figure SDC-2). Participants in the Strong underprediction condition were less certain than participants in the Correct prediction condition following the first post-suggestion trial and repetition of the suggestion.

Fear of upcoming pain. An exploration of fear of upcoming pain for all post-suggestion trials indicated main effects of condition, $F(1,77)=5.44, p=0.022, \eta_{G}{ }^{2}=0.06$, and trial, $F(4.32,332.52)=$ $9.41, p<0.001, \eta_{G}{ }^{2}=0.02$, reflecting continuously lower fear of pain in the Strong underprediction condition than in the Correct prediction condition (Figure SDC-3).

Pain unpleasantness. An exploration of pain unpleasantness for all post-suggestion trials indicated a main effect of trial, $F(3.97,309.86)=12.00, p<0.001, \eta_{G}{ }^{2}=0.02$, reflecting reductions in pain unpleasantness after the first trial in both conditions (Figure SDC-4).

\section{Autonomic responses}

There were no significantly differential effects of the strong underprediction versus correct prediction on heart rate, skin conductance level, or amplitude of the first skin conductance response (with regular or extended latency window) during the post-suggestion trials (Figures SDC-5 - SDC-8). There was a significant interaction effect for skin conductance level $F(3.28,249.33)=2.88, p=0.032$, $\eta_{G}{ }^{2}=0.01$, but no clearly distinct patterns were observed. A main effect of trial was observed for the skin conductance response with an extended latency window, $F(3.48,264.72)=5.16, p<0.001, \eta_{G}{ }^{2}=$ 0.04 , reflecting a decrease over time upon an initially strong response in both conditions.

\section{Psychological outcomes}

Descriptives (Table SDC-1) and between-group differences in awareness of the (mis)match of actual versus expected stimulus intensity, $F(1,78)=4.42, p=0.039, \eta_{G}{ }^{2}=0.05$, and of actual versus suggested stimulus intensity, $F(1,78)=31.34, p<0.001, \eta_{G}{ }^{2}=0.29$, indicated that participants were 
aware of the expectation/instruction-experience (mis)match. Notably, participants rated the trustworthiness of the experimenter who provided the verbal suggestion to be significantly lower in the Strong underprediction condition than in the Correct prediction condition, $F(1,78)=4.96, p=$ $0.029, \eta_{G}{ }^{2}=0.06$. No significantly differential effect of the suggestions on state anxiety was observed, nor where there significant between-group differences in the other measures (e.g., observation of (mis)match of the reference and post-suggestion trials, trustworthiness of the other experimenter, and response bias measures).

\section{Moderation by psychological characteristics}

The effect of the suggestions on experienced pain intensity during the first post-suggestion trial was significantly moderated by state anxiety, $b=-0.08, S E=0.03, t=-2.60, p=0.011$, reflecting that participants who reported lower state anxiety showed larger pain reductions upon the strong underprediction than those who reported higher state anxiety (pain intensity Strong underprediction condition at lower vs. upper quartile STAI-Ss $=5.5$ vs. 6.4). None of the other psychological characteristics (i.e., optimism, general trust, pain catastrophizing, ability to modify behavior, sensitivity to others, trait anxiety, or baseline fatigue) significantly moderated the effects.

\section{Study 2}

To better understand the effects of strongly underpredicting pain, we replicated Study 1 while adding a third experimental condition in which a medium underprediction of pain was given (Medium underprediction condition). This comparison allowed us, amongst others, to assess possibly reduced assimilation of experienced pain intensity upon strong underprediction (rather than contrast effects) and to compare the effects on trust. Furthermore, to increase the chances of contrast effects and to address new regulations, several changes were made in the experimental procedure and manipulations (e.g., increased intensity of heat pain stimuli).

\section{Methods Study 2}

\section{Participants}

As in Study 1, we included 41 healthy participants per condition, resulting in a total sample size of 123 participants. Eligibility criteria were the same as in Study 1.

\section{Procedure}

The experiment was conducted at the same location as Study 1, from March to October 2018. The study protocol was approved by Leiden University's Psychology Research Ethics Committee (CEP18-0306_133) and preregistered at the Netherlands Trial Register (NL7033).

The procedure was the same as for Study 1, with several exceptions. Participants now filled in the screening and other online questionnaires in the lab, in response to updates of European privacy regulations. Furthermore, the target temperature was set to now correspond to a high pain intensity rating of 7 to $\max 8$ on the pain intensity NRS $\left(M=48.4^{\circ} \mathrm{C}, S D=1.5^{\circ} \mathrm{C}\right)$ to increase the discrepancy between the predicted and actual stimulus intensity and thereby the chance of contrast effects. Also, the repeated presentations of the low and high stimulus were replaced by a single presentation of a high reference stimulus to simplify the comparison participants had to make and to minimize premanipulation exposure to correct instructions about stimulus intensity as this might also influence the 
likelihood of contrast effects. The thermode was now moved to a novel spot on the arm prior to the reference stimulus. Moreover, we now obtained ratings of expected pain intensity, certainty of pain expectation, fear of pain, and pain unpleasantness scores also for the reference stimulus to serve as comparators in our analyses. Medoc software issues were still present for many participants, stimuli were at peak for 4.0 to 10.4 seconds $(M=5.6, S D=2.1 \mathrm{sec}$ ). See Figure 3 for an overview of the procedure.

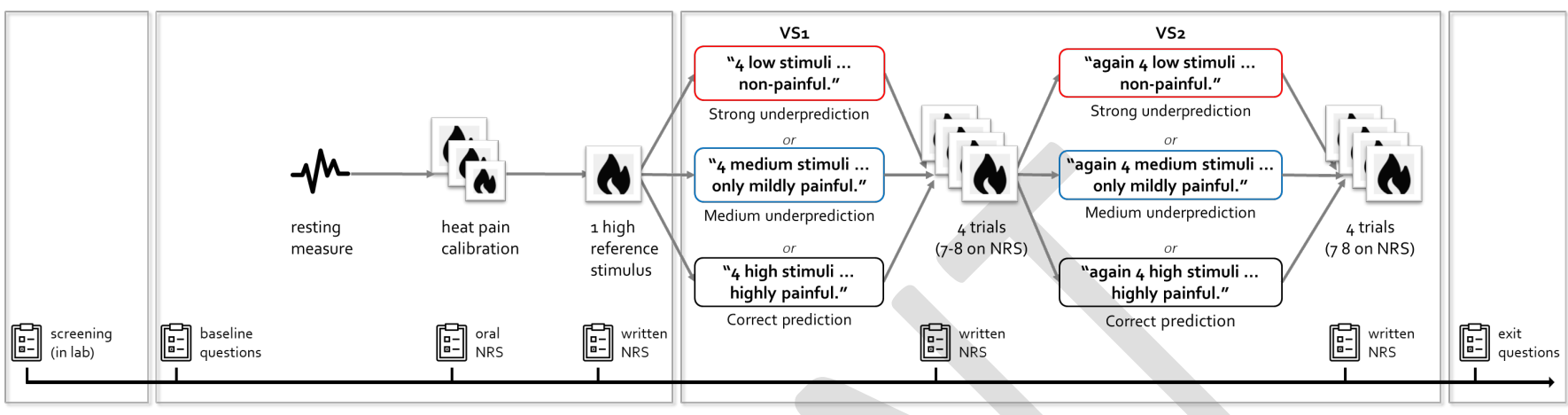

baseline

post-suggestion phase

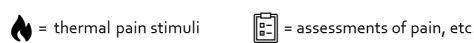

Figure 3.

Overview procedure with all measures taken in Study 2.

NRS = numerical ratings scale; VS = verbal suggestion

\section{Verbal suggestions of pain intensity}

The condition-specific verbal suggestions were slightly rephrased. Participants in the Strong underprediction condition were told "You'll now get 4 low stimuli. These are a lot lower than the reference stimulus. Based on your responses before, it is very likely that you will experience them as non-painful."

Participants in the Medium underprediction condition were told: "You'll now get 4 medium stimuli. These are noticeably lower than the reference stimulus. Based on your responses before, it is very likely that you will experience them as only mildly painful."

Participants in the Correct prediction condition were told: "You'll now get 4 high stimuli. These are equally high as the reference stimulus. Based on your responses before, it is very likely that you will experience them as highly painful."

The condition-specific suggestion was repeated before giving 4 additional trials: "You will now again get 4 low/medium/high stimuli. It is very likely that you'll experience them as non-painful / only mildly painful / highly painful."

\section{Measures}

The same pain rating scales, autonomic measures, and questionnaires were used as in Study 1. In addition, participants rated how competent, sympathetic, and honest they perceived the experimenters to be on 0-10 VAS. The questionnaires had acceptable to good internal consistency (LOT-R $\alpha=0.77$; GTS $\alpha=0.78$; PCS $\alpha=0.89$; SMS-R ability to modify self-presentation $\alpha=0.81$; SMS-R sensitivity to others $\alpha=0.78$; STAI-T $\alpha=0.91$; baseline STAI-Ss $\alpha=0.80$; post-intervention STAI-Ss $\alpha=$ 0.80 ). 


\section{Statistical analyses}

The same analysis strategy was used as in Study 1, with two deviations. First, because we now compared three instead of two conditions, omnibus AN(C)OVAs in which condition with 3 levels was an independent variable were followed up by AN(C)OVAs comparing the Strong underprediction condition with 1) the Correct prediction condition and 2) the Medium underprediction condition. For readability, only results of pairwise comparisons are reported, but only in cases of significant omnibus effects. Second, we now included ratings for the reference stimulus as a covariate for analyses of all pain outcomes.

\section{Results Study 2}

\section{Data availability}

Table SDC-2 presents the descriptives ( $M \pm S D$ ) per condition of all reported measures. The data are available via https://osf.io/24mu5/ (doi: 10.17605/OSF.IO/24MU5). [The final analysis scripts and results files will be uploaded upon publication of the paper in a peer-reviewed journal.]

\section{Participants}

Four participants were excluded during the screening in the lab. All 123 eligible participants completed participation ( $50.4 \%$ female; $\mathrm{M}$ age $=22.1$, SD $=2.9 ; 38.2 \%$ Dutch nationality; median baseline pain $=0.0$, interquartile range $=0.0$ ) .

\section{Effects of verbal suggestions on pain intensity}

Pairwise ANCOVAs indicated that the strong underprediction of pain led to significantly lower pain intensity of the first post-suggestion trial than the correct prediction, $F(1,79)=48.21, p<0.001$, $\eta_{G}{ }^{2}=0.38$, while it did not significantly differ from the medium underprediction, $F(1,79)=3.56, p=$ $0.063, \eta_{G}{ }^{2}=0.04$ (Figure $4 A$ ). Sensitivity analyses with stratification variable sex or stimulus peak duration as an additional covariate and without covariates yielded the same conclusions for the comparison of the Strong underprediction and Correct prediction conditions, but indicated significantly lower pain intensity after strong versus medium underprediction when stimulus peak duration was included as a covariate, $F(1,72)=4.54, p=0.037, \eta_{G}{ }^{2}=0.06$, or when no covariates where included, $F(1,80)=4.02, p=0.048, \eta_{G}{ }^{2}=0.05$.

An exploration of pain intensity during all post-suggestion trials, indicated main effects of condition, $F(1,79)=25.22, p<0.001, \eta_{G}{ }^{2}=0.20$, and trial, $F(3.34,264.15)=5.33, p<0.001, \eta_{G}{ }^{2}=$ 0.01 , for the comparison of the Strong underprediction and Correct prediction conditions, reflecting continuously lower pain in the Strong underprediction condition as compared to the Correct prediction condition and slight sensitization in these conditions. For the comparison of the Strong and Medium underprediction conditions, there was an interaction effect, $F(2.96,231.14)=3.13, p=0.027$, $\eta_{\mathrm{G}}{ }^{2}=0.01$, but no clearly distinct patterns were observed. 
A

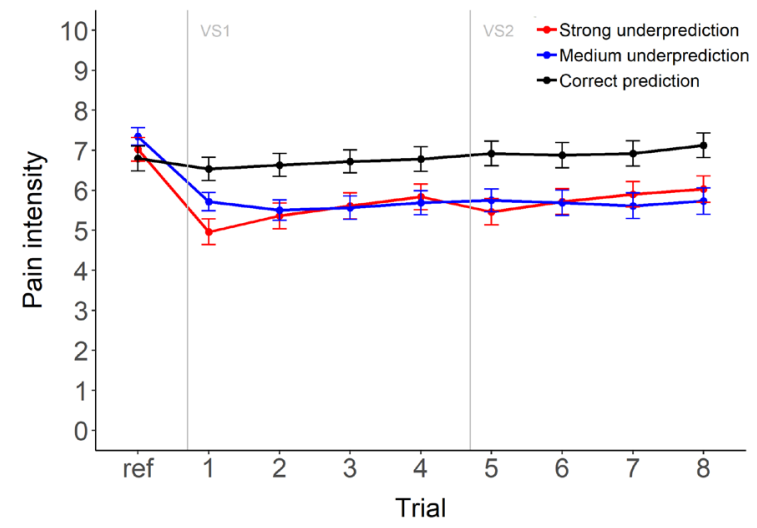

B

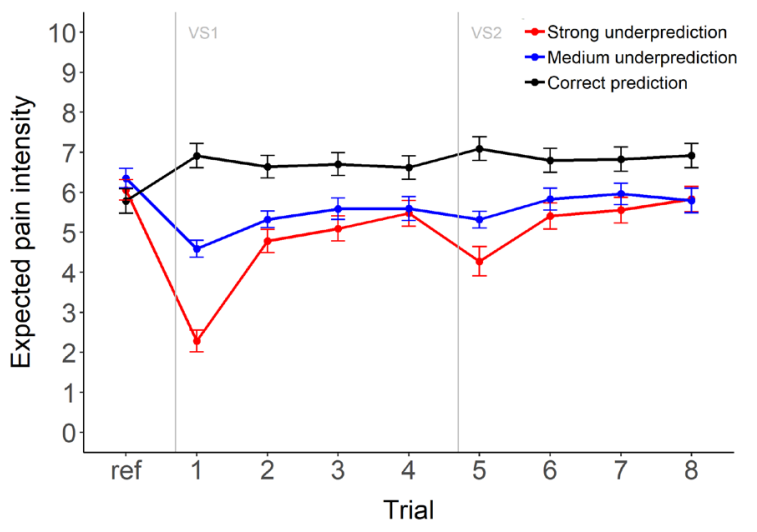

Figure 4.

A. Experienced pain intensity ratings $(M \pm S E)$ upon the reference stimulus and each of the 8 postsuggestion trials, and $B$. Expected pain intensity ratings $(M \pm S E$ ) prior to the reference stimulus and each of the 8 post-suggestion trials, per condition, in Study 2.

Ref = reference stimulus; VS = verbal suggestion.

\section{Expected pain intensity}

The verbal suggestions successfully manipulated expected pain intensity. Pairwise ANCOVAs indicated that the strong underprediction led participants to expect significantly less pain for the first post-suggestion trial than the correct prediction, $F(1,79)=243.55, p<0.001, \eta_{G}{ }^{2}=0.76$, and the medium underprediction, $F(1,79)=52.62, p<0.001, \eta_{G}{ }^{2}=0.40$ (Figure 4B).

Regarding trial 2, pairwise ANCOVAs indicated that participants in the Strong underprediction condition, who had experienced a violation of their initial expectation of low pain, also expected the following trial to be less painful than participants who received a correct prediction, $F(1,79)=57.00$, $\mathrm{p}<0.001, \mathrm{\eta}_{\mathrm{G}}{ }^{2}=0.42$, but not when compared to a medium underprediction.

An exploration of expected pain intensity during all trials, indicated a main effect of condition, $F(1,79)=72.83, p<0.001, \eta_{G}{ }^{2}=0.37$, and an interaction effect, $F(3.56,281.04)=33.66, p<0.001$, $\eta_{\mathrm{G}}{ }^{2}=0.14$, for the comparison of the Strong underprediction and Correct prediction conditions.

Participants in the Strong underprediction condition consistently expected less pain than participants in the Correct prediction condition, particularly directly upon the suggestions. For the comparison of the Strong and Medium underprediction conditions, there was an interaction effect, $F(4.12,321.31)=$ 11.06, $\mathrm{p}<0.001, \eta_{\mathrm{G}}{ }^{2}=0.06$, reflecting differences between the conditions directly upon the suggestions only.

\section{Other pain outcomes}

Certainty of pain expectation. A pairwise ANCOVA comparing certainty of pain expectations for all post-suggestion trials in the Strong underprediction and Correct prediction conditions indicated main effects of condition, $\mathrm{F}(1,79)=26.71, \mathrm{p}<0.001, \mathrm{\eta}_{\mathrm{G}}{ }^{2}=0.17$, and trial, $\mathrm{F}(4.73,373.61)=3.88, \mathrm{p}=$ $0.002, \eta_{G}{ }^{2}=0.02$, and an interaction effect, $F(4.73,373.61)=2.42, p=0.038, \eta_{G}{ }^{2}=0.01$ (Figure SDC9). Participants in the Strong underprediction condition were less certain than participants in the Correct prediction condition of their expectations across trials, particularly upon repetition of the verbal suggestion. For the comparison of the Strong and Medium underprediction conditions, a main effect of stimulus, $F(5.18,404.27)=6.00, p<0.001, \eta_{G}{ }^{2}=0.04$, and an interaction effect were 
observed, $F(5.18,404.27)=2.58, p=0.024, \eta_{G}{ }^{2}=0.01$, reflecting lower certainty only upon repetition of the strong underprediction suggestion.

Fear of upcoming pain. A pairwise ANCOVA comparing fear of upcoming pain for all postsuggestion trials in the Strong underprediction and Correct prediction conditions indicated a main effect of condition, $F(1,79)=14.14, p<0.001, \eta_{G}{ }^{2}=0.10$, and an interaction effect, $F(4.05,319.56)=$ $13.07, p<0.001, \eta_{G}{ }^{2}=0.06$ (Figure SDC-10). Participants in the Strong underprediction condition had less fear of pain for those trials closely following the suggestions than participants in the Correct prediction condition. For the comparison of the Strong and Medium underprediction conditions, an interaction effect was observed, $F(3.63,283.19)=2.93, p=0.025, \eta_{G}{ }^{2}=0.01$, reflecting lower fear upon strong underprediction for the first stimulus only.

Pain unpleasantness. A pairwise ANCOVA comparing pain unpleasantness for all postsuggestion trials in the Strong underprediction and Correct prediction conditions indicated main effects of condition, $F(1,79)=7.42, p=0.008, \eta_{G}{ }^{2}=0.06$, and trial, $F(4.14,327.22)=2.75, p=0.027$, $\eta_{\mathrm{G}}{ }^{2}=0.01$, reflecting continuously lower pain unpleasantness in the Strong underprediction condition as compared to the Correct prediction condition, and a slight increase of pain unpleasantness over time in both groups (Figure SDC-11). For the comparison of the Strong and Medium underprediction conditions, an interaction effect was observed, $F(3.78,295.02)=3.04, p=0.020, \eta_{G}{ }^{2}=0.01$, reflecting slight increases in pain unpleasantness over trials in the Strong underprediction condition only.

\section{Autonomic responses}

Heart rate. A pairwise ANCOVA comparing heart rate for all post-suggestion trials in the Strong underprediction and Correct prediction conditions indicated an interaction effect, $F(7,483)=5.75, p<$ $0.001, \eta_{G}{ }^{2}=0.02$ (Figure SDC-12), reflecting that participants in the Strong underprediction condition had a lower heart rate than participants in the Correct prediction condition during the first trials. For the comparison of the Strong and Medium underprediction conditions, a main effect of condition was observed, $F(1,74)=6.29, p=0.014, \eta_{G}{ }^{2}=0.06$, reflecting continuously lower heart rate upon the strong underprediction as compared to the medium underprediction.

Skin conductance level. A pairwise ANCOVA comparing skin conductance level for all postsuggestion trials in the Strong underprediction and Correct prediction conditions indicated an interaction effect, $F(3.11,217.97)=10.31, p<0.001, \eta_{G}{ }^{2}=0.02$ (Figure SDC-13), reflecting that participants in the Strong underprediction condition had a lower skin conductance level than participants in the Correct prediction condition during the first trial only. For the comparison of the Strong and Medium underprediction conditions, no significant effects were observed.

Skin conductance response. Pairwise ANCOVAs comparing the amplitude of the first skin conductance response for all post-suggestion trials indicated main effects of condition for the comparisons of the Strong underprediction with both the Correct prediction condition and the Medium underprediction condition $\left(F(1,71)=5.46, p=0.022, \eta_{G}{ }^{2}=0.03\right.$ and $F(1,73)=10.57, p=$ $0.002, \eta_{G}{ }^{2}=0.05$, respectively), reflecting continuously higher responses in the Strong underprediction condition than in the other conditions (Figure SDC-14). Sensitivity analyses using the extended latency windows confirmed the main effect of condition for the comparison of the Strong and Medium underprediction conditions only, $F(1,74)=11.42, p=0.001, \eta_{G}{ }^{2}=0.09$, while also showing a main effect of trial, $F(5.7,421.49)=2.38, p=0.031, \eta_{G}{ }^{2}=0.01$, reflecting a slight decrease over time in these conditions (Figure SDC-15). 


\section{Psychological outcomes}

Descriptives (Table SDC-2) and pairwise ANOVAs indicated that participants were aware of the condition-dependent instruction-experience (mis)match (strong underprediction vs. correct prediction: $F(1,80)=34.64, p<0.001, \eta_{G}{ }^{2}=0.30$; strong vs. medium underprediction: $F(1,80)=$ $13.52, p<0.001, \eta_{G}{ }^{2}=0.14$ ). This was also largely supported by the perceived (mis)matches of expected and experienced pain, although there was no significant difference between the strong underprediction and correct prediction (strong underprediction vs. correct prediction: $F(1,80)=1.33$, $p=0.252, \eta_{G}{ }^{2}=0.02$; strong vs. medium underprediction: $F(1,80)=6.78, p=0.011, \eta_{G}{ }^{2}=0.08$ ).

Notably, pairwise ANOVAs indicated that trustworthiness of the experimenter who provided the verbal suggestion was rated significantly lower upon a strong underprediction than upon a correct prediction, $\mathrm{F}(1,80)=14.96, \mathrm{p}<0.001, \mathrm{n}_{\mathrm{G}}{ }^{2}=0.16$, but not as compared to a medium underprediction. In addition, pairwise ANOVAs indicated that honesty of this experimenter was rated lower upon a strong underprediction than upon either a correct prediction, $F(1,80)=32.24, p<$ $0.001, \eta_{G}{ }^{2}=0.29$, or a medium underprediction, $F(1,80)=4.86, p=0.030, \eta_{G}{ }^{2}=0.06$.

Last, pairwise ANOVAs indicated that participants rated a higher sense of owing it to the experimenter to report less pain in the Strong underprediction condition as compared to the Correct prediction condition, $F(1,80)=11.44, p=0.001, \eta_{G}{ }^{2}=0.13$. No significantly differential effect of the suggestions on state anxiety was observed, nor were there significant between-group differences in the other measures (e.g., observation of discrepancy between reference and post-suggestion trials, trust in the other experimenter, perception of experimenters as competent and sympathetic, and other response bias measures).

\section{Moderation by psychological characteristics}

Comparisons of the Strong underprediction and Correct prediction conditions, indicated that the effect of the suggestions on experienced pain intensity during the first post-suggestion trial was significantly moderated by dispositional optimism, $b=-0.17, \mathrm{SE}=0.06, \mathrm{t}=-2.71, \mathrm{p}=0.008$, reflecting that participants who reported lower optimism showed larger pain reductions upon the strong underprediction than those who reported higher optimism (pain intensity Strong underprediction condition at lower vs. upper quartile LOT-R $=4.5$ vs. 5.3). In line, a significant moderation by trait pain catastrophizing, $b=0.07, \mathrm{SE}=0.03, \mathrm{t}=2.28, \mathrm{p}=0.025$, reflected that participants who reported higher catastrophizing showed larger pain reductions upon the strong underprediction than those who reported low catastrophizing (pain intensity Strong underprediction condition at lower vs. upper quartile PCS $=5.3$ vs. 4.6). Furthermore, but in contrast to the finding of Study 1 , a significant moderation by state anxiety, $b=0.05, S E=0.03, t=2.05, p=0.043$, reflected that participants who reported higher state anxiety showed larger pain reductions upon the strong underprediction than those who reported low state anxiety (pain intensity Strong underprediction condition at lower vs. upper quartile STAI-Ss $=5.2$ vs. 4.7). No other moderations were observed.

\section{Discussion}

We investigated the effects of strong underpredictions of pain. Contrary to our hypothesis, results of both studies showed that strong underpredictions of pain reduced experienced pain intensity. In other words, despite large expectation-experience discrepancies, assimilation of pain experiences to expectations occurred instead of contrast effects. Notably, Study 2 indicated that pain intensity reductions upon strong underpredictions were not considerably larger than upon medium underpredictions. Also expected pain, fear of pain, and pain unpleasantness were generally found to 
assimilate to the strong underpredictions of pain. Study 2 , in which a slightly larger and more noticeable expectation-experience discrepancy was used than in Study 1, further provided some indications, though inconsistent, for reduced heart rate and skin conductance levels (i.e., assimilation), but increased skin conductance responses (i.e., contrast effects). Finally, aside from these generally positive findings, we observed important counteractive effects of strong underpredictions of pain, namely increased uncertainty and lowered trust in the person providing the instructions, though again not considerably more so than medium underpredictions.

Participants of both studies who were given a verbal suggestion that subsequent heat stimuli would be non-painful, which in fact were moderately (Study 1) or highly (Study 2) painful, reported substantially less intense pain than participants who were correctly informed about the stimulus intensity. This assimilation of pain experiences to the expectation was observed for a first heat pain trial directly following the underprediction and was maintained for subsequent trials, also upon repetition of the suggestion. These findings are in line with the vast majority of literature on expectancy and placebo effects demonstrating expectations to generally be self-confirming $[2,3,8,10,31,32,40,43-45,54,57]$. However, they contradict our hypothesis of contrasts effects (i.e., increased pain), which we formulated based on the literature on the occurrence of contrast effects on diverse outcomes in cases of large expectation-experience discrepancies of which one is aware $[1,7,11,18,19,33,40,41,57]$. A lack of awareness of the discrepancy is unlikely to explain our current findings, as we intentionally drew participants' attention to the expectation-experience (mis)match. Participants' resultant awareness was indicated by how they compared their experiences with what they expected and were instructed. Nonetheless, it might be that their focus on their experiences made participants aware of the ambiguous nature of pain and thereby actually facilitated assimilation $[10,17]$. As such, it appears that assimilation of pain experiences to expectations is the rule.

Notably, Study 2 showed no reliably significant differences in pain intensity upon a strong versus medium underprediction of pain intensity. This finding complements a study demonstrating smaller placebo effects of decaffeinated coffee on alertness upon suggestions of high versus moderate caffeine doses [33] and recent experiments demonstrating a tipping point at which large expectation-experience discrepancies led to less assimilation of pain experiences than moderate discrepancies [24]. Thus, there might be a limit to the effects of underpredicting pain intensity in terms of the benefits for pain relief.

We conducted secondary and several exploratory analyses to gain a broader understanding of the effects of strongly underpredicting pain, beyond the effects on experienced pain intensity. Both studies demonstrated that also expected pain intensity, fear of pain, and pain unpleasantness generally reduced, i.e., assimilated, upon a strong underprediction as compared to a correct prediction. Again, Study 2 demonstrated that the strong underprediction generally exerted no larger effects than the medium underprediction. Assimilation of these pain responses is in line with the larger placebo literature [43], but contradicts a study in which underprediction of pain led to increased expectations and fear of pain [3]. Aside from these arguable positive effects of underpredicting pain, it should be noted that participants were also mostly less certain of their expectations upon strong underprediction, particularly in Study 2, but not more so than upon a medium underprediction. Uncertainty is an outcome that is generally overlooked (but see [3]), but may importantly lower the impact of future suggestions by rendering them as less convincing and thereby reducing their influence on future outcomes [10,30]. Note, however, that participants in our studies continued to assimilate their experienced pain intensity when the underprediction was 
repeated for a second series of trials, despite apparent reduced effects of the suggestion on expectations.

Examinations of autonomic responses to the heat stimuli indicated no significant effects of the suggestions in Study 1, but Study 2 provided some indications for lower heart rate and skin conductance level during few early trials upon the strong underprediction as compared to the correct prediction (i.e., assimilation effects). In contrast, there were some indications, though inconsistent, for increased skin conductance responses upon the strong underprediction as compared to the correct prediction and medium underprediction (i.e., contrast effects). Interpretation of these findings is hampered by their inconsistency, high variability, and relatively weak responses to the heat stimuli as compared to rest (see Tables SDC-1 and SDC-2), but they suggest that effects of the verbal suggestions may go beyond self-report effects. Confidence in this interpretation is strengthened by the general absence of evidence for between-group differences in response bias measures and moderation of the effects of pain underpredictions on pain intensity by participants' ability to modify self-presentation and sensitivity to expressive behaviors of others. Moreover, the observed psychophysiological responses might indicate that different measures of autonomic responses can highlight different underlying processes. One might for example speculate that upon an initial startled response when pain is higher than expected, responses are regulated so that on average, even over the course of seconds, autonomic responses align with self-reported pain reductions, if any response is visible at all. Findings of previous research are also largely equivocal, with some providing evidence for placebo responses on heart rate $[6,46]$ and skin conductance responses [15,21,39], while other studies showed no significant effects (heart rate: $[4,20,38]$, skin conductance response: [29]).

Further explorations of participants' responses to the underprediction of pain led to the novel and potentially highly clinically relevant finding that despite assimilation of pain, even upon repetition of the suggestion, participants reported substantially lower trustworthiness of the experimenter who provided the suggestion upon strong underprediction as compared to correct prediction of pain, and not considerably more than upon medium underprediction. Thus, there appears to be a trade-off between reducing pain and fostering trust. Given that trust is critical in good clinical communication and is also thought to be key in placebo effects [22], one might advocate for providing honest, correct information about upcoming pain (e.g., during a medical procedure), even when short-term pain relief opportunities might then be missed. However, future research replicating these findings, also in clinical contexts, examining long-term consequences, and examining other factors that might be at play (e.g., characteristics of the interpersonal relationship like communicated warmth and competence), is required before conclusions can be drawn and clearcut advice for clinical implementation can be formulated.

Last, we examined if individual differences in initial responses to strong underpredictions of pain could be explained by several trait and state personal characteristics. We found some indications for moderation by state anxiety, optimism, and pain catastrophizing of the effects of the strong underprediction versus correct prediction, but the effects were small, inconsistent, and - in the case of state anxiety - contradictory. Hence, adding to previous literature $[25,44,55]$, it might be inferred that no consistent, readily identifiable profile of responders to suggestions exists.

When considering the implications of the current findings for clinical practice, it should be kept in mind that the current studies were conducted with verbal suggestions about brief heat stimuli in healthy samples. This allows for stringent experimental control of stimulus intensity and participants' expectations about the stimuli, but hampers translation to clinical practice. Previous findings do 
suggest that experimental placebo studies with acute experimental pain translate well to acute clinical pain, but this appears less so for chronic pain outcomes [43], and this might not apply for salient expectation-experience mismatches. Moreover, trust in an experimenter might more readily be affected by a simple suggestion than trust in a healthcare provider, especially when vulnerability and hence the need for trust is high and/or when a long-established trusting relationship is established [22]. On the other hand, violations of trust might lead to particularly strong responses, like a sense of betrayal, when personal relevance is high. Future research should examine the studied effects in more clinically relevant contexts, including long-term outcomes. Moreover, the effects might differ depending on the ambiguity of the expectations and sensations $[7,10,30]$, the direction of the mismatch (underpredicting pain may have a larger impact than overprediction [3]), attention and affect $[19,26]$, and prior experiences [18]. Also, effects might differ in cases that expectations do not directly pertain to the intensity of the pain stimulus (i.e., stimulus expectations) but to the effects of (placebo) treatments on pain (i.e., treatment outcome expectations) [50].

In conclusion, even strong underpredictions of pain can reduce pain, although not considerably more so than medium underpredictions. However, such overly positive instructions may cause uncertainty and undermine trust. Thus, underpredicting pain has both benefits and risks. Although further research is required, these findings suggest that healthcare providers may wish to be cautious with providing overly positive information about painful medical procedures, despite pain-relieving effects.

\section{Acknowledgements}

We are indebted to Dr. A.I.M. van Laarhoven and Prof. A.W.M. Evers for their support in setting up the research. We are thankful to all students who helped with data-collection and processing of the data when working on their bachelor thesis, master thesis, or research internship for their essential input and dedication. Moreover, we thank all study participants.

All authors have nothing to disclose.

The research was preregistered in the Netherlands Trial Register (Study 1: NL6228, Study 2: NL7033). The data are available via https://osf.io/24mu5/ (doi: 10.17605/OSF.IO/24MU5). [The final analysis scripts and results files will be uploaded upon publication of the paper in a peer-reviewed journal.]

\section{References}

[1] Anderson RE. Consumer Dissatisfaction: The Effect of Disconfirmed Expectancy on Perceived Product Performance. J Mark Res 1973;10:38.

[2] Arntz A, Hopmans M. Underpredicted pain disrupts more than correctly predicted pain, but does not hurt more. Behav Res Ther 1998;36:1121-1129.

[3] Arntz A, van den Hout MA, van den Berg G, Meijboom A. The effects of incorrect pain expectations on acquired fear and pain responses. Behav Res Ther 1991;29:547-560.

[4] Aslaksen PM, Flaten MA. The Roles of Physiological and Subjective Stress in the Effectiveness of a Placebo on Experimentally Induced Pain: Psychosom Med 2008;70:811-818.

[5] Benedetti F. Placebo effects. Second edition. Oxford, UK: Oxford University Press, 2014 p. 
[6] Benedetti F, Arduino C, Costa S, Vighetti S, Tarenzi L, Rainero I, Asteggiano G. Loss of expectation-related mechanisms in Alzheimer's disease makes analgesic therapies less effective: Pain 2006;121:133-144.

[7] Bless H, Burger AM. Assimilation and contrast in social priming. Curr Opin Psychol 2016;12:2631.

[8] Bless H, Schwarz N. Mental Construal and the Emergence of Assimilation and Contrast Effects: The Inclusion/Exclusion Model. Adv Exp Soc Psychol 2010;42:319-373.

[9] Borm GF, Fransen J. A simple sample size formula for analysis of covariance in randomized clinical trials. J Clin Epidemiol 2007:5.

[10] Büchel C, Geuter S, Sprenger C, Eippert F. Placebo Analgesia: A Predictive Coding Perspective. Neuron 2014;81:1223-1239.

[11] Buckingham G. Getting a grip on heaviness perception: a review of weight illusions and their probable causes. Exp Brain Res 2014;232:1623-1629.

[12] Cacioppo JT, Tassinary LG, Berntson G. Handbook of Psychophysiology. Cambridge University Press, $2007 \mathrm{p}$.

[13] Campbell C, Guy A. 'Why Can't They Do Anything for a Simple Back Problem?': A Qualitative Examination of Expectations for Low Back Pain Treatment and Outcome. J Health Psychol 2007;12:641-652.

[14] Colagiuri B, Schenk LA, Kessler MD, Dorsey SG, Colloca L. The placebo effect: From concepts to genes. Neuroscience 2015;307:171-190.

[15] Eippert F, Bingel U, Schoell ED, Yacubian J, Klinger R, Lorenz J, Büchel C. Activation of the Opioidergic Descending Pain Control System Underlies Placebo Analgesia. Neuron 2009;63:533-543.

[16] Evers AWM, Colloca L, Blease C, Annoni M, Atlas LY, Benedetti F, Bingel U, Büchel C, Carvalho C, Colagiuri B, Crum AJ, Enck P, Gaab J, Geers AL, Howick J, Jensen KB, Kirsch I, Meissner K, Napadow V, Peerdeman KJ, Raz A, Rief W, Vase L, Wager TD, Wampold BE, Weimer K, Wiech K, Kaptchuk TJ, Klinger R, Kelley JM. Implications of Placebo and Nocebo Effects for Clinical Practice: Expert Consensus. Psychother Psychosom 2018;87:204-210.

[17] Geers AL, Helfer SG, Weiland PE, Kosbab K. Expectations and Placebo Response: A Laboratory Investigation into the Role of Somatic Focus. J Behav Med 2006;29:171-178.

[18] Geers AL, Lassiter GD. Affective Assimilation and Contrast: Effects of Expectations and Prior Stimulus Exposure. Basic Appl Soc Psychol 2005;27:143-154.

[19] Geers AL, Lassiter GD. Affective Expectations and Information Gain: Evidence for Assimilation and Contrast Effects in Affective Experience. J Exp Soc Psychol 1999;35:394-413.

[20] Geers AL, Wellman JA, Fowler SL, Helfer SG, France CR. Dispositional Optimism Predicts Placebo Analgesia. J Pain 2010;11:1165-1171.

[21] Geuter S, Eippert F, Hindi Attar C, Büchel C. Cortical and subcortical responses to high and low effective placebo treatments. Neurolmage 2013;67:227-236.

[22] Hall MA, Dugan E, Zheng B, Mishra AK. Trust in Physicians and Medical Institutions: What Is It, Can It Be Measured, and Does It Matter? Milbank Q 2001;79:613-639.

[23] Herruer JM, Prins JB, van Heerbeek N, Verhage-Damen GWJA, Ingels KJAO. Negative Predictors for Satisfaction in Patients Seeking Facial Cosmetic Surgery: A Systematic Review. Plast Reconstr Surg 2015;135:1596-1605.

[24] Hird EJ, Charalambous C, El-Deredy W, Jones AK, Talmi D. Boundary effects of expectation in human pain perception. Sci Rep 2019;9:9443.

[25] Horing B, Weimer K, Muth ER, Enck P. Prediction of placebo responses: a systematic review of the literature. Front Psychol 2014;5. doi:10.3389/fpsyg.2014.01079.

[26] Huntsinger JR. A Flexible Impact of Affective Feelings on Priming Effects: Assimilation and Contrast. Pers Soc Psychol Bull 2014;40:450-462.

[27] Husain A, Lee G-C. Establishing Realistic Patient Expectations Following Total Knee Arthroplasty. J Am Acad Orthop Surg 2015;23:707-713.

[28] Joffily M. EDA Toolbox. n.d. p. Available: https://github.com/mateusjoffily/EDA/wiki. 
[29] Jong PJ, Baast R, Arntz A, Merckelbach $\mathrm{H}$. The placebo effect in pain reduction: The influence of conditioning experiences and response expectancies. Int J Behav Med 1996;3:14-29.

[30] Kirsch I. Response Expectancy and the Placebo Effect. International Review of Neurobiology. Elsevier, 2018, Vol. 138. pp. 81-93. doi:10.1016/bs.irn.2018.01.003.

[31] Kirsch I. Response Expectancy as a Determinant of Experience and Behavior. Am Psychol 1985:14.

[32] Kirsch I. Response expectancy theory and application: A decennial review. Appl Prev Psychol 1997;6:69-79.

[33] Kirsch I, Weixel L. Double-Blind Versus Deceptive Administration of a Placebo. Behav Neurosci 1988;102:319-323.

[34] Lakens D. Calculating and reporting effect sizes to facilitate cumulative science: a practical primer for t-tests and ANOVAs. Front Psychol n.d.:12.

[35] Lasselin J, Petrovic P, Olsson MJ, Paues Göranson S, Lekander M, Jensen KB, Axelsson J. Sickness behavior is not all about the immune response: Possible roles of expectations and prediction errors in the worry of being sick. Brain Behav Immun 2018;74:213-221.

[36] Lennox RD, Wolfe RN. Revision of the Self-Monitoring Scale. J Pers Soc Psychol 1984;46:16.

[37] Marteau TM, Bekker $\mathrm{H}$. The development of a six-item short-form of the state scale of the Spielberger State-Trait Anxiety Inventory (STAI). Br J Clin Psychol 1992;31:301-306.

[38] Matre D. Placebo-Induced Changes in Spinal Cord Pain Processing. J Neurosci 2006;26:559-563.

[39] Nakamura Y, Donaldson GW, Kuhn R, Bradshaw DH, Jacobson RC, Chapman RC. Investigating dose-dependent effects of placebo analgesia: A psychophysiological approach: Pain 2012;153:227-237.

[40] Okamoto M, Dan I. Extrinsic information influences taste and flavor perception: A review from psychological and neuroimaging perspectives. Semin Cell Dev Biol 2013;24:247-255.

[41] Parker S, Moore JM, Bahraini S, Gunthert K, Zellner DA. Effects of expectations on loudness and loudness difference. Atten Percept Psychophys 2012;74:1334-1342.

[42] Peerdeman KJ, van Laarhoven AIM, Bartels DJP, Peters ML, Evers AWM. Placebo-like analgesia via response imagery. Eur J Pain 2017;21:1366-1377.

[43] Peerdeman KJ, van Laarhoven AIM, Keij SM, Vase L, Rovers MM, Peters ML, Evers AWM. Relieving patients' pain with expectation interventions: a meta-analysis. PAIN 2016;157:11791191.

[44] Peerdeman KJ, van Laarhoven AIM van, Peters ML, Evers AWM. An Integrative Review of the Influence of Expectancies on Pain. Front Psychol 2016;7. doi:10.3389/fpsyg.2016.01270.

[45] Petersen GL, Finnerup NB, Colloca L, Amanzio M, Price DD, Jensen TS, Vase L. The magnitude of nocebo effects in pain: A meta-analysis. Pain 2014;155:1426-1434.

[46] Pollo A, Vighetti S, Rainero I, Benedetti F. Placebo analgesia and the heart. Pain 2003;102:125133.

[47] Price DD, McGrath PA, Rafii A, Buckingham B. The validation of visual analogue scales as ratio scale measures for chronic and experimental pain: Pain 1983;17:45-56.

[48] Rolke R, Baron R, Maier C, Tölle TR, Treede - D. R., Beyer A, Binder A, Birbaumer N, Birklein F, Bötefür IC, Braune S, Flor H, Huge V, Klug R, Landwehrmeyer GB, Magerl W, Maihöfner C, Rolko C, Schaub C, Scherens A, Sprenger T, Valet M, Wasserka B. Quantitative sensory testing in the German Research Network on Neuropathic Pain (DFNS): Standardized protocol and reference values: Pain 2006;123:231-243.

[49] Scheier MF, Carver CS, Bridges MW. Distinguishing Optimism From Neuroticism (and Trait Anxiety, Self-Mastery, and Self-Esteem): A Reevaluation of the Life Orientation Test. J Pers Soc Psychol 1994;67:1063-1078.

[50] Schenk LA, Sprenger C, Onat S, Colloca L, Büchel C. Suppression of Striatal Prediction Errors by the Prefrontal Cortex in Placebo Hypoalgesia. J Neurosci 2017;37:9715-9723.

[51] Sjak-Shie EE. PhysioData Toolbox (version 0.5.0). 2019 p. Available: https://PhysioDataToolbox.leidenuniv.nl. 
[52] Spielberger CD, Gorsuch RL. State-trait anxiety inventory for adults: Manual and sample: Manual, instrument and scoring guide. Consulting Psychologists Press, $1983 \mathrm{p}$.

[53] Sullivan MJL, Bishop SR, Pivik J. The Pain Catastrophizing Scale: Development and Validation. Psychol Assess 1995;7.

[54] Vase L, Petersen GL, Riley JL, Price DD. Factors contributing to large analgesic effects in placebo mechanism studies conducted between 2002 and 2007: Pain 2009;145:36-44.

[55] Weimer K, Colloca L, Enck P. Placebo effects in psychiatry: mediators and moderators. Lancet Psychiatry 2015;2:246-257.

[56] Welty LJ, Rasmussen LV, Baldridge AS, Whitley E. StatTag. Chicago, Illinois, United States: Galter Health Sciences Library, 2016 p. doi:10.18131/G36K76.

[57] Wilson TD, Lisle DJ, Kraft D, Wetzel CG. Preferences as Expectation-Driven Inferences: Effects of Affective Expectations on Affective Experience. J Pers Soc Psychol 1989;56:12.

[58] Yamagishi T, Yamagishi M. Trust and commitment in the United States and Japan. Motiv Emot 1994;18:129-166. 


\section{Supplemental Digital Content}

Table SDC-1. Means and standard deviations for all relevant measures in Study 1

\begin{tabular}{|c|c|c|c|}
\hline Measure & Condition & $\begin{array}{c}\text { Strong } \\
\text { underprediction } \\
(n=40)\end{array}$ & $\begin{array}{c}\text { Moderate } \\
\text { underprediction } \\
(n=41)\end{array}$ \\
\hline \multicolumn{4}{|l|}{ Sample characteristics } \\
\hline Age & & $23.2 \pm 2.6$ & $22.6 \pm 2.8$ \\
\hline Sex ( $N, \%$ female) & & $20,50 \%$ & $21,51 \%$ \\
\hline Nationality (N, \% Dutch) & & $20,50 \%$ & $21,51 \%$ \\
\hline Baseline pain & & $0.2 \pm 0.4$ & $0.3 \pm 0.6$ \\
\hline Baseline fatigue & & $3.1 \pm 2.2$ & $3.2 \pm 2.3$ \\
\hline \multicolumn{4}{|l|}{ Pain outcomes } \\
\hline Temperature high \& test stimuli $\left({ }^{\circ} \mathrm{C}\right)$ & & $47.4 \pm 2.1$ & $47.4 \pm 1.7$ \\
\hline \multicolumn{4}{|l|}{ Pain expectation a } \\
\hline Prior to test stimulus 1 & & $1.4 \pm 2.1$ & $5.6 \pm 2.1$ \\
\hline Prior to test stimulus 2 & & $4.9 \pm 2.2$ & $6.6 \pm 1.8$ \\
\hline Prior to test stimulus 3 & & $5.2 \pm 1.8$ & $6.2 \pm 2.0$ \\
\hline Prior to test stimulus 4 & & $5.2 \pm 1.9$ & $5.9 \pm 1.9$ \\
\hline Prior to test stimulus 5 & & $3.4 \pm 2.1$ & $6.3 \pm 2.0$ \\
\hline Prior to test stimulus 6 & & $5.1 \pm 2.0$ & $6.3 \pm 1.8$ \\
\hline Prior to test stimulus 7 & & $5.4 \pm 1.8$ & $6.3 \pm 2.3$ \\
\hline Prior to test stimulus 8 & & $5.5 \pm 1.9$ & $6.1 \pm 2.3$ \\
\hline \multicolumn{4}{|l|}{ Certainty of expectation ${ }^{a}$} \\
\hline Prior to test stimulus 1 & & $7.4 \pm 2.4$ & $6.2 \pm 2.7$ \\
\hline Prior to test stimulus 2 & & $5.0 \pm 2.5$ & $6.6 \pm 2.4$ \\
\hline Prior to test stimulus 3 & & $5.5 \pm 2.5$ & $6.6 \pm 2.4$ \\
\hline Prior to test stimulus 4 & & $5.8 \pm 2.7$ & $6.2 \pm 2.7$ \\
\hline Prior to test stimulus 5 & & $4.4 \pm 2.6$ & $6.9 \pm 2.1$ \\
\hline Prior to test stimulus 6 & & $5.7 \pm 2.8$ & $6.7 \pm 2.8$ \\
\hline Prior to test stimulus 7 & & $6.1 \pm 2.7$ & $6.6 \pm 2.3$ \\
\hline Prior to test stimulus 8 & & $6.0 \pm 2.8$ & $7.0 \pm 2.1$ \\
\hline \multicolumn{4}{|l|}{ Fear of pain a } \\
\hline Prior to test stimulus 1 & & $1.0 \pm 1.6$ & $2.7 \pm 2.3$ \\
\hline Prior to test stimulus 2 & & $2.6 \pm 2.6$ & $3.7 \pm 2.6$ \\
\hline Prior to test stimulus 3 & & $2.1 \pm 2.2$ & $3.0 \pm 2.7$ \\
\hline Prior to test stimulus 4 & & $2.0 \pm 2.2$ & $3.0 \pm 2.8$ \\
\hline Prior to test stimulus 5 & & $1.6 \pm 1.9$ & $3.3 \pm 2.6$ \\
\hline Prior to test stimulus 6 & & $1.9 \pm 1.9$ & $2.9 \pm 2.7$ \\
\hline Prior to test stimulus 7 & & $1.8 \pm 1.7$ & $2.7 \pm 2.7$ \\
\hline Prior to test stimulus 8 & & $1.8 \pm 2.0$ & $2.7 \pm 2.8$ \\
\hline \multicolumn{4}{|l|}{ Pain intensity a } \\
\hline High reference stimuli (average) & & $6.7 \pm 0.9$ & $6.4 \pm 1.4$ \\
\hline Test stimulus 1 & & $6.1 \pm 1.7$ & $7.0 \pm 1.5$ \\
\hline Test stimulus 2 & & $5.6 \pm 1.6$ & $6.4 \pm 1.6$ \\
\hline Test stimulus 3 & & $5.6 \pm 1.6$ & $6.2 \pm 1.8$ \\
\hline Test stimulus 4 & & $5.7 \pm 1.7$ & $6.3 \pm 1.8$ \\
\hline Test stimulus 5 & & $5.3 \pm 1.9$ & $6.2 \pm 1.9$ \\
\hline Test stimulus 6 & & $5.5 \pm 1.7$ & $6.3 \pm 2.0$ \\
\hline Test stimulus 7 & & $5.6 \pm 1.8$ & $6.3 \pm 2.1$ \\
\hline Test stimulus 8 & & $5.7 \pm 1.9$ & $6.3 \pm 2.3$ \\
\hline \multicolumn{4}{|l|}{ Pain unpleasantness a } \\
\hline Test stimulus 1 & & $5.4 \pm 2.0$ & $6.5 \pm 2.1$ \\
\hline Test stimulus 2 & & $4.3 \pm 1.9$ & $5.3 \pm 2.7$ \\
\hline Test stimulus 3 & & $4.4 \pm 2.0$ & $5.0 \pm 3.0$ \\
\hline Test stimulus 4 & & $4.3 \pm 2.0$ & $5.2 \pm 3.3$ \\
\hline Test stimulus 5 & & $4.4 \pm 2.1$ & $5.3 \pm 3.1$ \\
\hline Test stimulus 6 & & $4.5 \pm 2.1$ & $5.1 \pm 3.2$ \\
\hline Test stimulus 7 & & $4.6 \pm 2.3$ & $5.1 \pm 3.2$ \\
\hline
\end{tabular}




\section{Autonomic responses}

Heart rate

Rest

$77.1 \pm 12.4$

$74.3 \pm 11.4$

High reference stimuli (average)

$77.3 \pm 12.2$

$74.8 \pm 10.7$

Test stimulus 1

$75.9 \pm 12.7$

$74.8 \pm 11.1$

Test stimulus 2

$76.2 \pm 11.3$

$73.9 \pm 10.6$

Test stimulus 3

$75.8 \pm 12.1$

$73.2 \pm 11.2$

Test stimulus 4

$75.4 \pm 11.9$

$74.1 \pm 11.5$

Test stimulus 5

$74.6 \pm 12.1$

$73.1 \pm 10.1$

Test stimulus 6

$74.8 \pm 11.8$

$74.0 \pm 10.8$

Test stimulus 7

$76.1 \pm 11.0$

$74.0 \pm 11.0$

Test stimulus 8

$74.9 \pm 11.1$

$73.1 \pm 9.5$

Skin conductance level

Rest

High reference stimuli (average)

$4.6 \pm 2.9$

$4.7 \pm 2.6$

Test stimulus 1

$7.6 \pm 2.9$

$7.6 \pm 2.8$

$7.7 \pm 2.9$

Test stimulus 2

$7.3 \pm 2.8$

Test stimulus 3

$7.1 \pm 2.8$

Test stimulus 4

$7.0 \pm 2.8$

$8.1 \pm 3.1$

$7.5 \pm 2.8$

$7.1 \pm 2.7$

$6.9 \pm 2.7$

$6.8 \pm 2.6$

$7.2 \pm 2.8$

Test stimulus 5

$6.8 \pm 2.6$

Test stimulus 7

$6.7 \pm 2.6$

$7.0 \pm 2.7$

$6.8 \pm 2.7$

Test stimulus 8

$6.6 \pm 2.6$

Skin conductance response (amplitude first response)

Rest

High reference stimuli (average)

$0.1 \pm 0.2$

$6.8 \pm 2.7$

Test stimulus 1

$0.2 \pm 0.2$

$0.3 \pm 0.4$

Test stimulus 2

Test stimulus 3

$0.3 \pm 0.3$

$0.2 \pm 0.3$

$0.2 \pm 0.3$

est stimulus 4

$0.2 \pm 0.3$

$0.2 \pm 0.3$

Test stimulus 6

$0.2 \pm 0.2$

Test stimulus 7

$0.2 \pm 0.2$

$0.2 \pm 0.3$

$0.3 \pm 0.4$

$0.3 \pm 0.4$

$0.2 \pm 0.3$

$0.2 \pm 0.2$

$0.2 \pm 0.3$

$0.3 \pm 0.4$

$0.3 \pm 0.4$

$0.2 \pm 0.3$

Test stimulus 8

$0.2 \pm 0.4$

Skin conductance response (amplitude first response, extended latency window)

Rest

$0.1 \pm 0.2$

$0.2 \pm 0.3$

High reference stimuli (average)

$0.4 \pm 0.4$

$0.5 \pm 0.4$

$1.1 \pm 0.9$

$1.3 \pm 1.0$

Test stimulus 2

$0.4 \pm 0.4$

$0.5 \pm 0.6$

Test stimulus 3

$0.3 \pm 0.3$

$0.3 \pm 0.3$

Test stimulus 4

$0.3 \pm 0.4$

$0.3 \pm 0.4$

Test stimulus 5

$0.3 \pm 0.4$

$0.4 \pm 0.4$

Test stimulus 6

$0.3 \pm 0.6$

$0.3 \pm 0.3$

$0.2 \pm 0.3$

Test stimulus 8

$0.2 \pm 0.2$

$0.2 \pm 0.4$

Psychological outcomes

STAI-Ss - state anxiety (post)

$31.8 \pm 8.8$

$31.8 \pm 9.2$

Previous experience with heat pain tests

$1.1 \pm 0.5$

$1.2 \pm 0.6$

$7.6 \pm 1.5$

$4.4 \pm 2.2$

Pain intensity during previous heat pain test $(n=7)$

$8.3 \pm 1.5$

$8.6 \pm 1.5$

Idea of study purpose ${ }^{b}$

$3.3 \pm 2.5$

$3.0 \pm 2.5$

$8.1 \pm 2.1$

$8.1 \pm 1.4$

$5.9 \pm 1.9$

Pain test vs high reference stimuli $c$

$5.6 \pm 1.6$

$5.4 \pm 2.2$

Actual vs expected pain test stimuli ${ }^{c}$

$6.5 \pm 2.2$

$5.1 \pm 1.8$

$7.6 \pm 2.1$

$7.7 \pm 2.1$

Trustworthiness experimenter $A^{a}$

$6.8 \pm 2.6$

$6.9 \pm 2.5$

(who gave verbal suggestions)

Concerned what experimenter thought ${ }^{b}$

$3.3 \pm 3.2$

$2.6 \pm 2.6$ 
Changed responses to help experimenter ${ }^{b}$ Owed it to experimenter to report less or more pain ${ }^{c}$ Provided answers experimenter wanted to hear ${ }^{b}$

\section{Psychological characteristics}

LOT-R - optimism

GTS - general trust

PCS - pain catastrophizing

SMS-R ability to modify self-presentation

SMS-R sensitivity to others

STAI-T - trait anxiety

STAI-Ss- state anxiety (baseline)

$\begin{array}{ll}1.4 \pm 2.3 & 1.2 \pm 1.8 \\ 4.3 \pm 1.6 & 4.7 \pm 1.1 \\ 1.8 \pm 2.6 & 1.6 \pm 2.1\end{array}$

$16.1 \pm 3.2$

$15.8 \pm 3.4$

$4.6 \pm 1.0$

$4.5 \pm 1.2$

$13.7 \pm 8.7$

$14.3 \pm 8.3$

$22.8 \pm 4.4$

$22.0 \pm 4.3$

$20.3 \pm 3.5$

$20.2 \pm 3.5$

$37.9 \pm 8.7$

$36.5 \pm 6.9$

$32.0 \pm 8.5$

Note. Means and standard deviations $(M \pm S D)$ are presented for all available data. ${ }^{a}=$ range 0 ( $n o(t)$ at all) - 10 (most imaginable); ${ }^{b}=\operatorname{range} 0$ (not at all) -10 (very $($ much $\left.)\right){ }^{c}=\operatorname{range} 0$ (much less) -5 (equal) -10 (much more). 


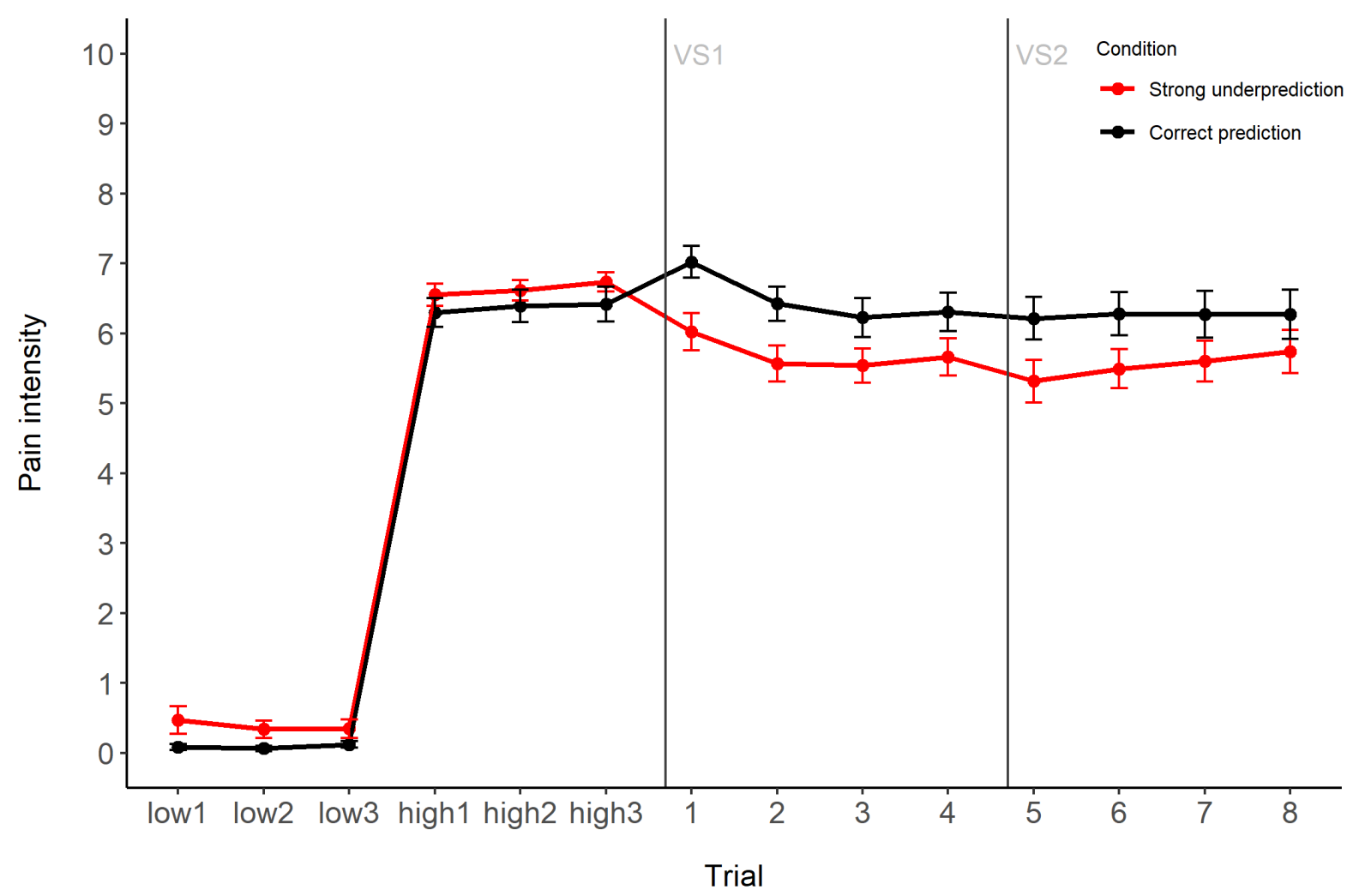

Figure SDC-1.

Pain intensity ratings (mean \pm standard error) for the 3 low reference stimuli, 3 high reference stimuli, and the 8 test stimuli per condition in Study 1.

Low $=$ low reference stimulus; high = high reference stimulus; VS = verbal suggestion. 


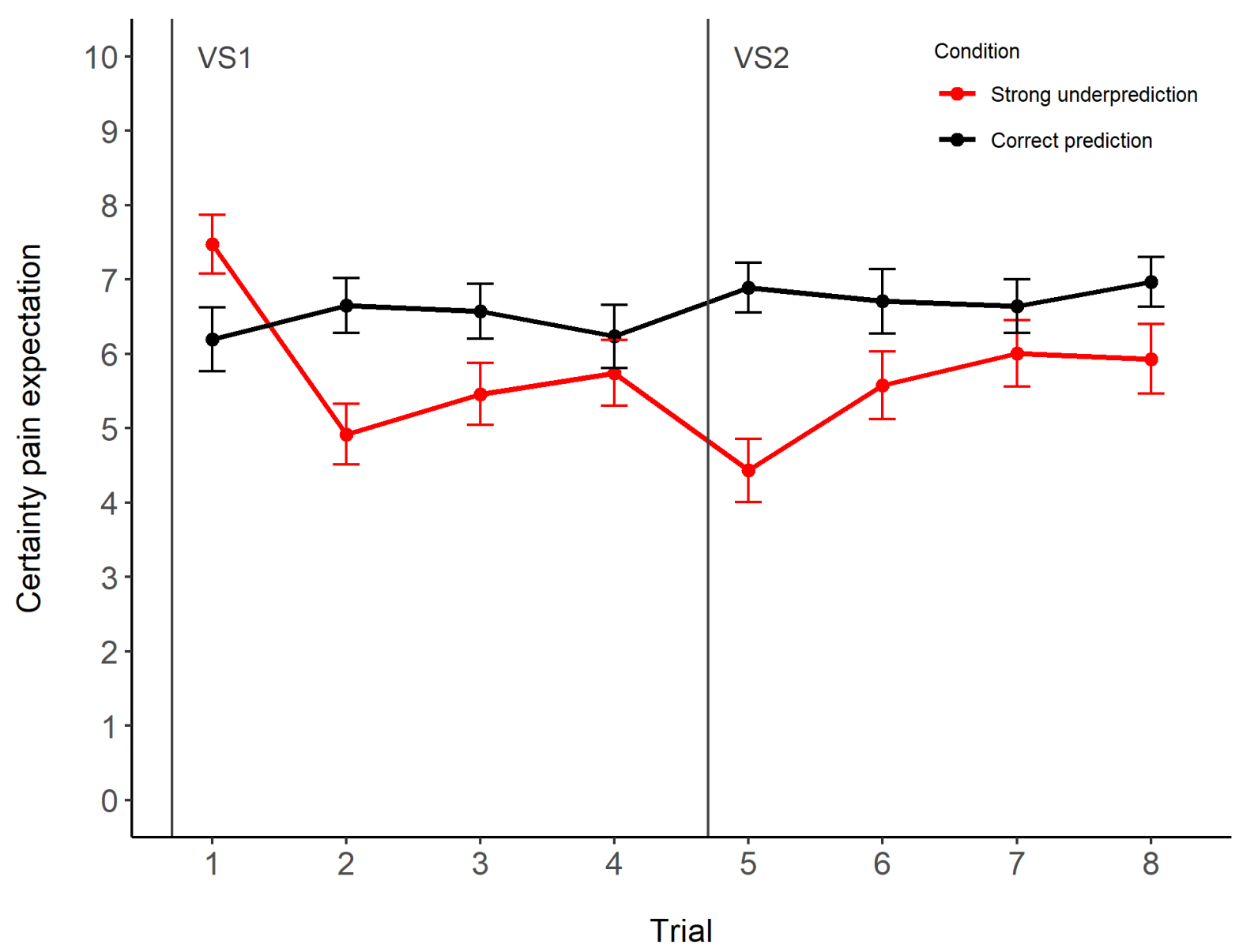

Figure SDC-2.

Certainty of pain expectation ratings (mean \pm standard error) for the 8 test stimuli per condition in Study 1.

VS = verbal suggestion . 


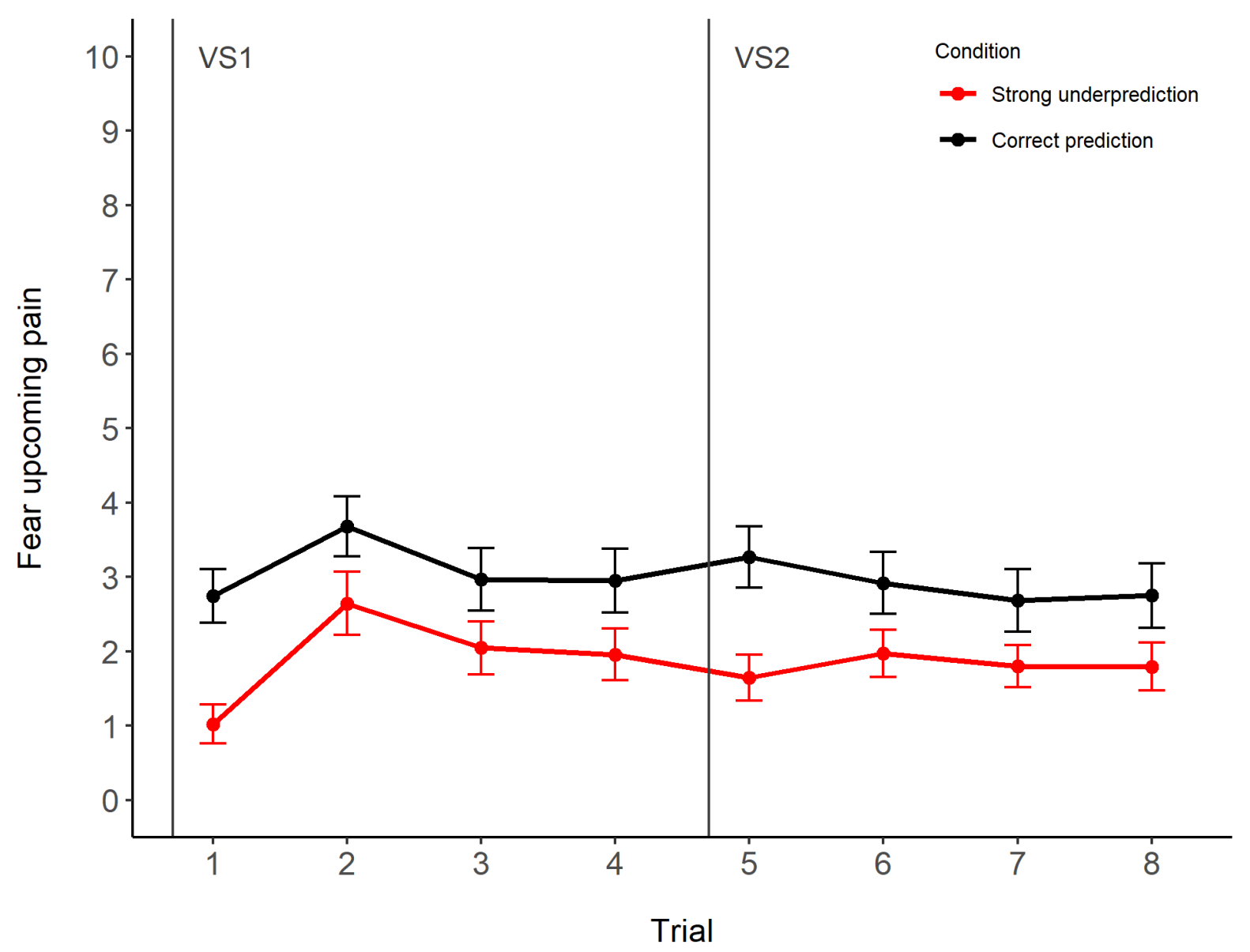

Figure SDC-3.

Fear of upcoming pain ratings (mean \pm standard error) for the 8 test stimuli per condition in Study 1 . VS = verbal suggestion 


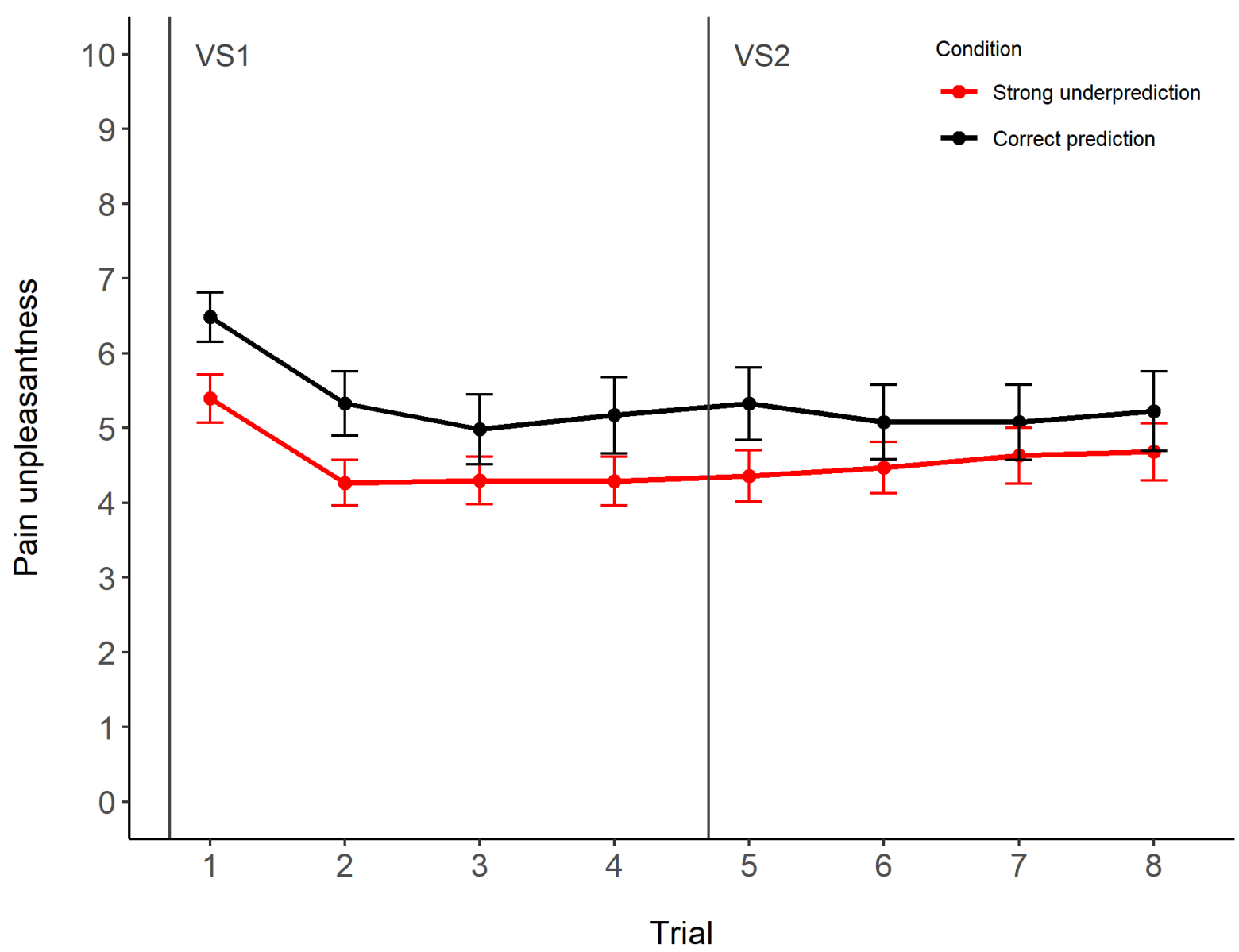

Figure SDC-4.

Pain unpleasantness ratings (mean \pm standard error) for the 8 test stimuli per condition in Study 1. VS = verbal suggestion. 


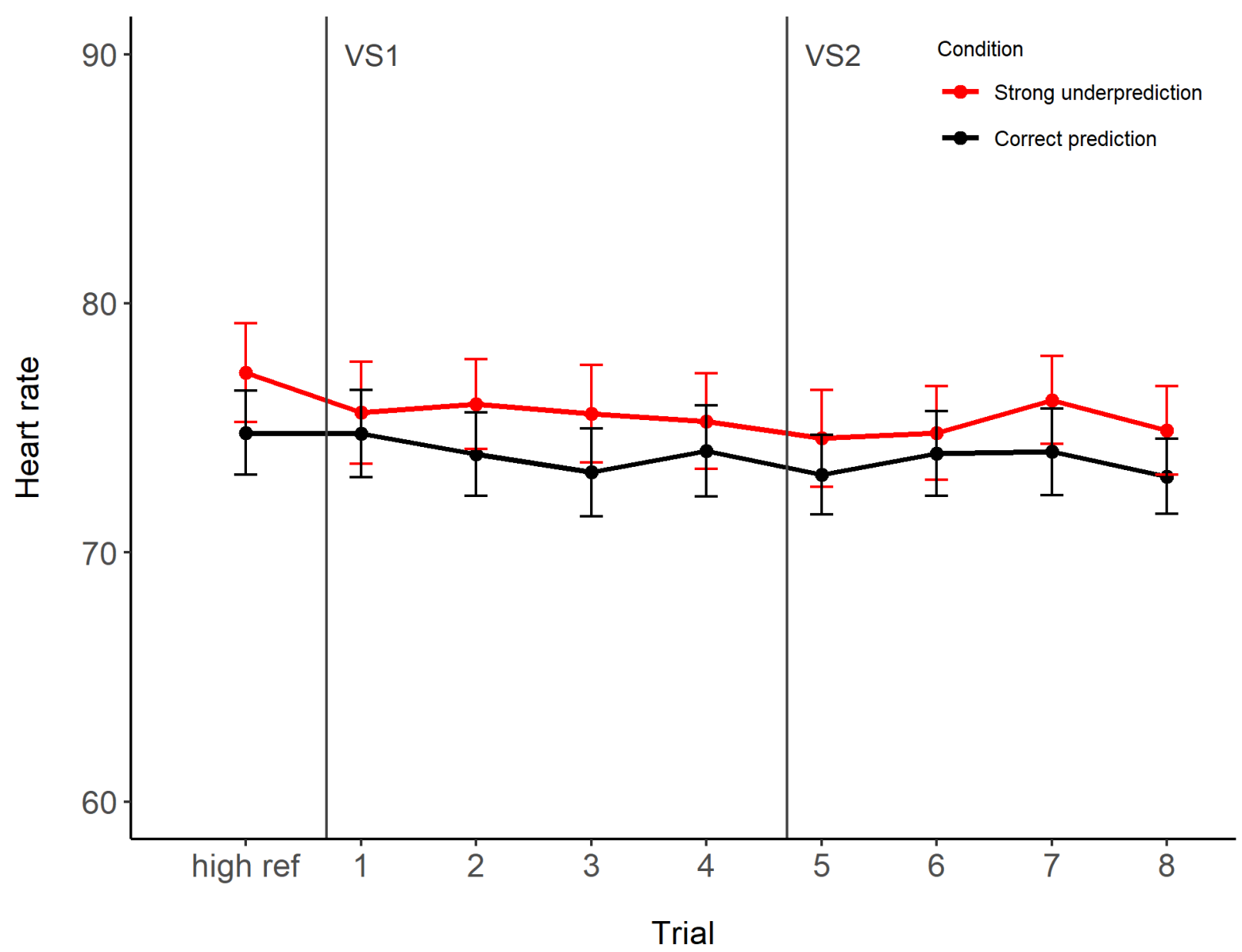

Figure SDC-5.

Heart rate (mean \pm standard error) for the 3 high reference stimuli (average) and the 8 test stimuli per condition in Study 1.

High ref = average of the 3 high reference trials; VS = verbal suggestion. 


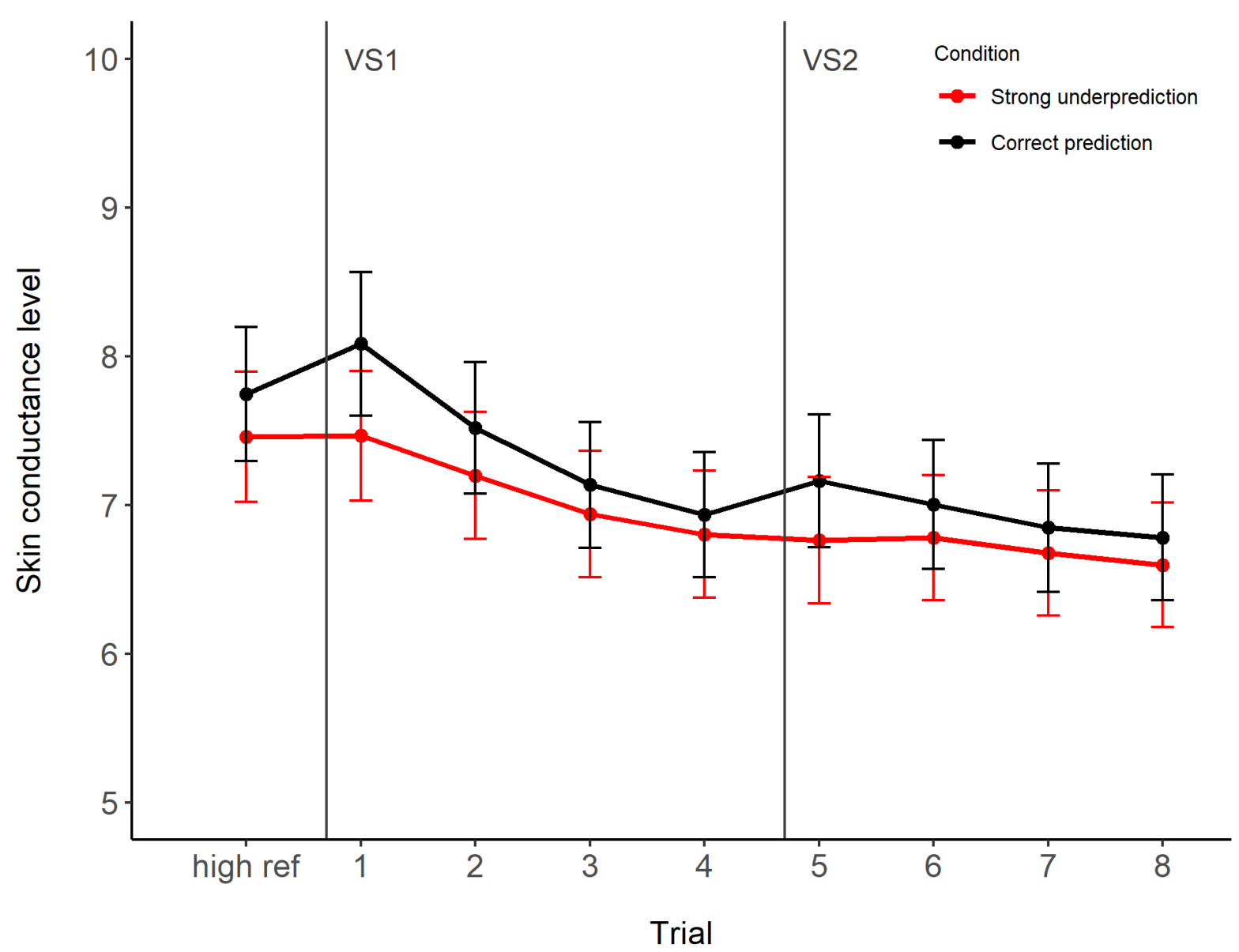

Figure SDC-6.

Skin conductance level (mean \pm standard error) for the 3 high reference stimuli (average) and the 8 test stimuli per condition in Study 1.

High ref = average of the 3 high reference trials; VS = verbal suggestion. 


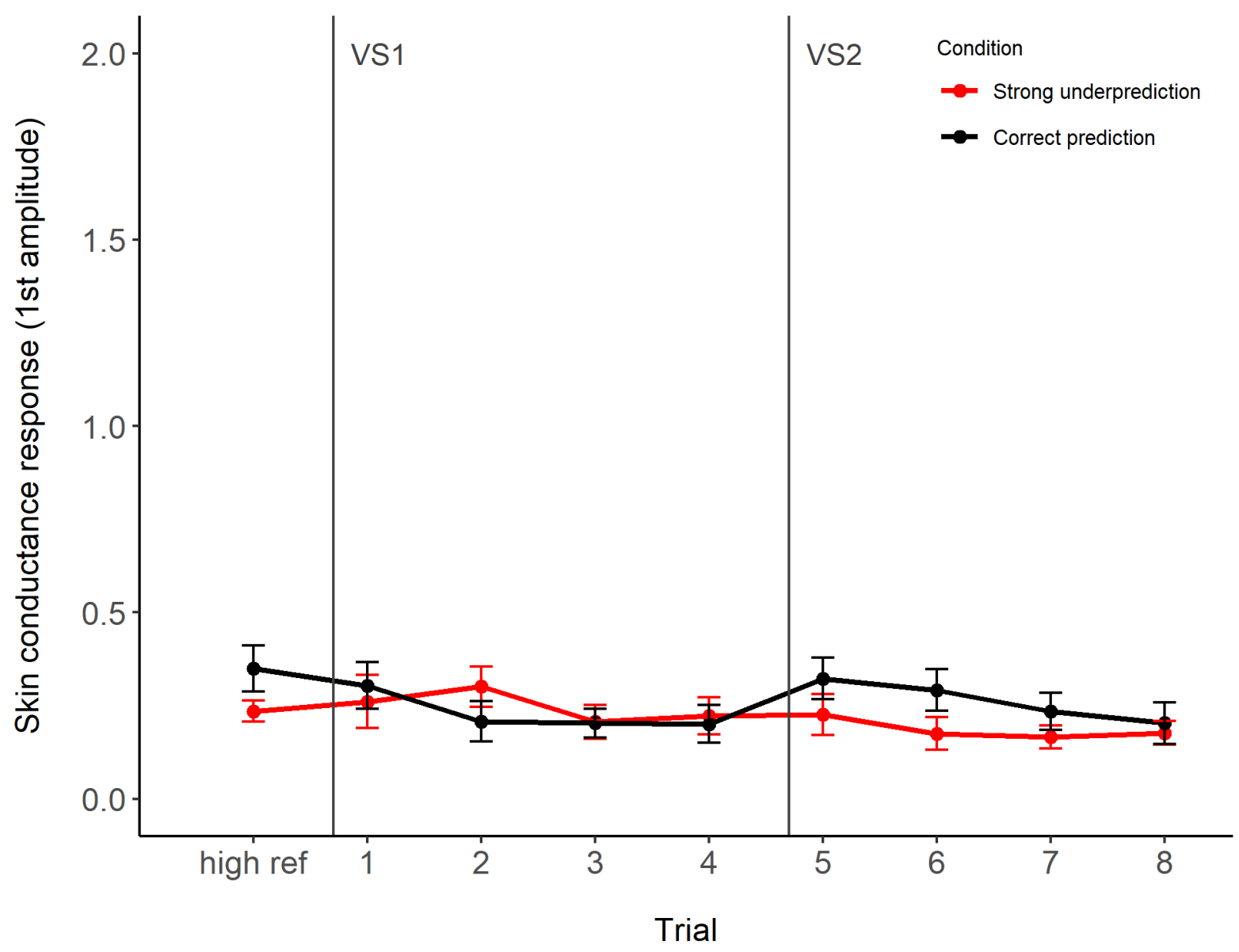

Figure SDC-7.

Amplitude first skin conductance response (mean \pm standard error) for the 3 high reference stimuli (average) and the 8 test stimuli per condition in Study 1.

High ref $=$ average of the 3 high reference trials; VS = verbal suggestion. 


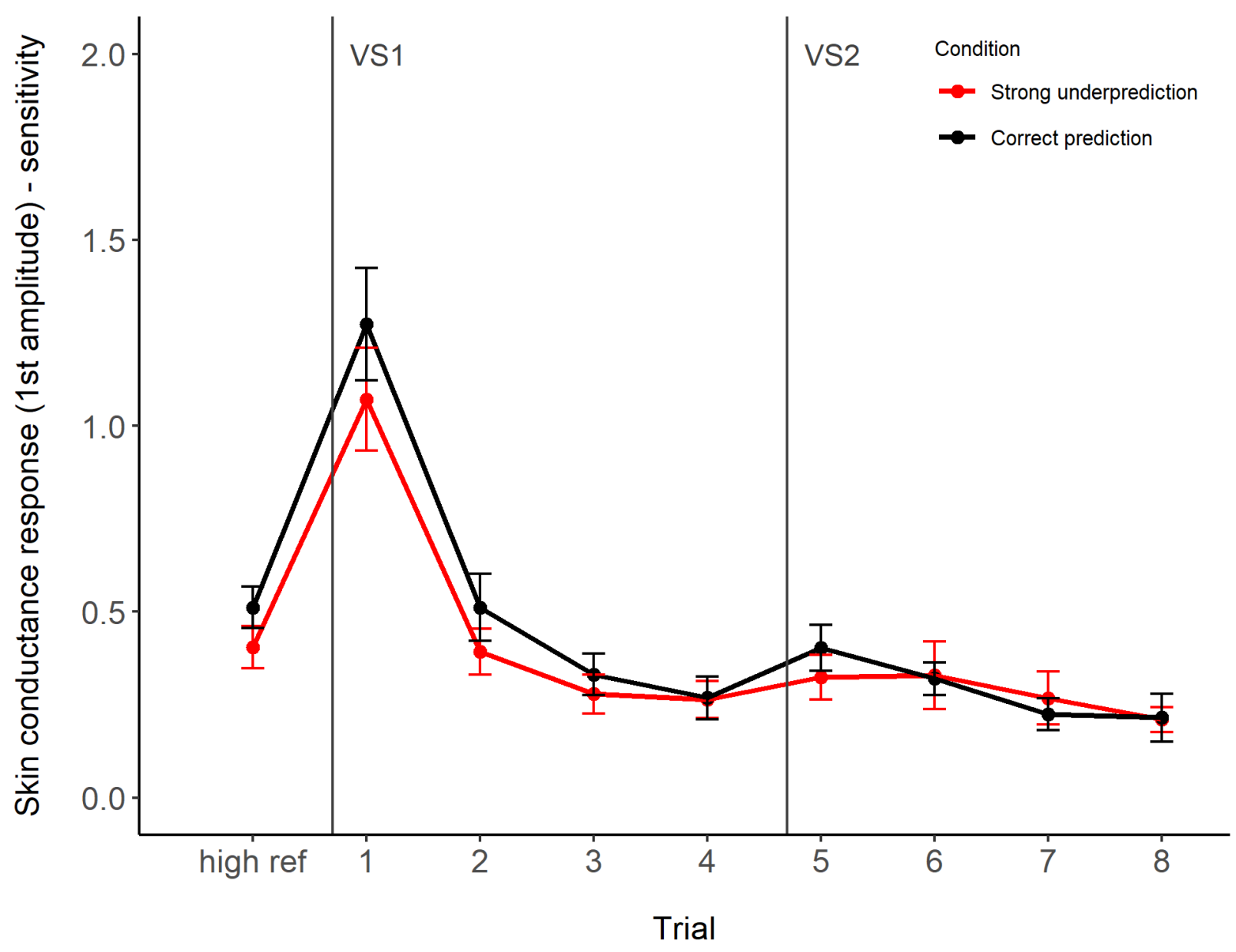

\section{Figure SDC-8.}

Amplitude first skin conductance response, with extended latency window (mean \pm standard error) for the 3 high reference stimuli (average) and the 8 test stimuli per condition in Study 1.

High ref = average of the 3 high reference trials; VS = verbal suggestion. 
Table SDC-2. Means and standard deviations for all relevant measures in Study 2

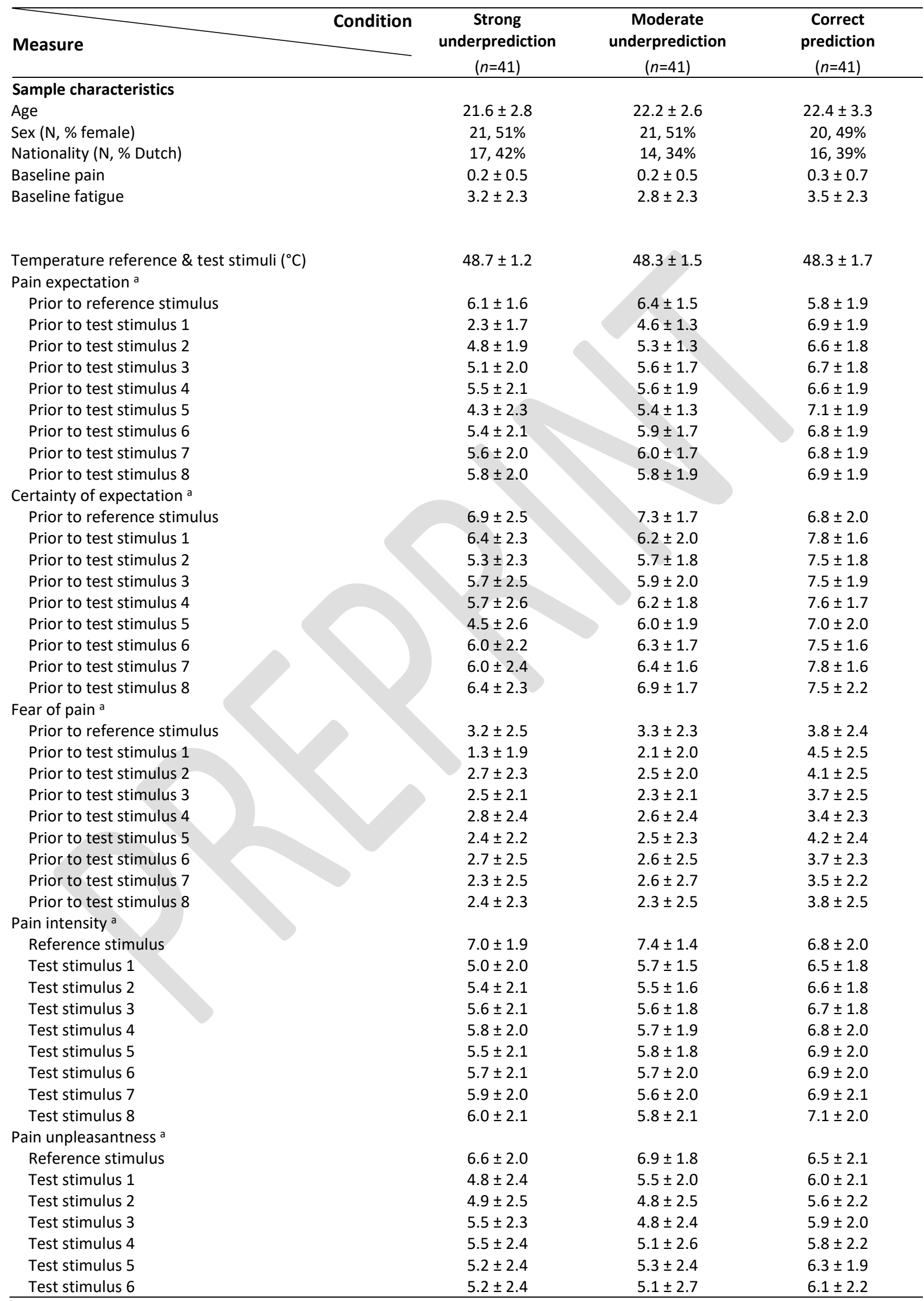




\begin{tabular}{llll}
\hline Test stimulus 7 & $5.3 \pm 2.6$ & $4.8 \pm 2.7$ & $6.3 \pm 2.1$ \\
Test stimulus 8 & $5.5 \pm 2.8$ & $5.0 \pm 2.8$ & $6.4 \pm 2.1$
\end{tabular}

\section{Autonomic responses} Heart rate

Rest

Reference stimulus

Test stimulus 1

Test stimulus 2

Test stimulus 3

Test stimulus 4

Test stimulus 5

Test stimulus 6

Test stimulus 7

Test stimulus 8

Skin conductance level

Rest

Reference stimulus

Test stimulus 1

Test stimulus 2

Test stimulus 3

Test stimulus 4

Test stimulus 5

Test stimulus 6

Test stimulus 7

Test stimulus 8

Skin conductance response (amplitude first response)

Rest

Reference stimulus

Test stimulus 1

Test stimulus 2

Test stimulus 3

Test stimulus 4

Test stimulus 5

Test stimulus 6

Test stimulus 7

Test stimulus 8

Skin conductance response (amplitude first response,

extended latency window)

Rest

Reference stimulus

Test stimulus 1

Test stimulus 2

Test stimulus 3

Test stimulus 4

Test stimulus 5

Test stimulus 6

Test stimulus 7

Test stimulus 8

\section{Psychological outcomes}

STAI-Ss - state anxiety (post)

Previous experience with heat pain tests

Pain intensity during previous heat pain test $(n=2)$

Focus on sensations during tests ${ }^{b}$

Idea of study purpose ${ }^{b}$

Pain test vs reference stimulus ${ }^{c}$

Actual vs expected pain test stimuli ${ }^{c}$

Actual vs instructed pain test stimuli ${ }^{c}$

Trustworthiness experimenter $A^{a}$

Competent $^{a}$

Sympathetic a

Honest $^{a}$
$77.5 \pm 12.0$
$80.4 \pm 11.4$
$73.1 \pm 10.3$
$75.7 \pm 9.9$
$76.3 \pm 10.1$
$75.3 \pm 9.4$
$74.7 \pm 9.9$
$75.7 \pm 10.0$
$75.4 \pm 10.2$
$76.4 \pm 11.3$

$5.4 \pm 2.6$

$8.7 \pm 3.8$

$7.4 \pm 3.3$

$7.5 \pm 3.4$

$7.4 \pm 3.4$

$7.3 \pm 3.3$

$7.2 \pm 3.3$

$7.3 \pm 3.5$

$7.2 \pm 3.4$

$7.0 \pm 3.3$

$0.4 \pm 0.5$

$0.2 \pm 0.4$

$0.6 \pm 0.7$

$0.6 \pm 0.7$

$0.5 \pm 0.6$

$0.4 \pm 0.5$

$0.6 \pm 0.7$

$0.5 \pm 0.6$

$0.6 \pm 0.6$

$0.5 \pm 0.6$

$0.4 \pm 0.5$

$1.5 \pm 1.3$

$1.0 \pm 0.9$

$0.9 \pm 0.9$

$0.9 \pm 0.9$

$0.8 \pm 0.8$

$0.9 \pm 1.0$

$0.9 \pm 0.9$

$0.7 \pm 0.8$

$0.8 \pm 0.8$

$35.7 \pm 10.4$

$1.1 \pm 0.4$

$8.1 \pm 1.6$

$8.4 \pm 1.5$

$3.1 \pm 2.4$

$5.9 \pm 2.1$

$6.8 \pm 2.2$

$7.5 \pm 2.2$

$7.4 \pm 2.1$

$7.8 \pm 1.8$

$7.5 \pm 1.8$

$7.5 \pm 2.0$

$\begin{array}{ll}77.2 \pm 12.2 & 78.1 \pm 12.1 \\ 77.6 \pm 12.0 & 79.1 \pm 14.2 \\ 74.1 \pm 10.8 & 77.9 \pm 13.9 \\ 76.5 \pm 11.3 & 78.5 \pm 12.3 \\ 75.7 \pm 11.5 & 74.5 \pm 13.3 \\ 76.2 \pm 11.9 & 75.2 \pm 13.0 \\ 75.4 \pm 11.3 & 76.5 \pm 12.6 \\ 77.1 \pm 12.1 & 77.0 \pm 13.8 \\ 77.1 \pm 11.4 & 75.7 \pm 12.5 \\ 77.3 \pm 12.3 & 76.0 \pm 11.9\end{array}$

$5.1 \pm 2.6 \quad 4.8 \pm 2.5$

$8.1 \pm 2.9 \quad 8.4 \pm 3.5$

$6.8 \pm 2.6 \quad 7.8 \pm 3.0$

$7.0 \pm 2.6 \quad 7.4 \pm 2.9$

$6.8 \pm 2.5 \quad 7.1 \pm 2.8$

$6.8 \pm 2.6 \quad 6.9 \pm 2.7$

$6.7 \pm 2.5 \quad 7.1 \pm 2.9$

$6.7 \pm 2.5 \quad 7.0 \pm 2.8$

$6.6 \pm 2.5 \quad 6.7 \pm 2.7$

$6.6 \pm 2.6 \quad 6.7 \pm 2.7$

$0.2 \pm 0.2 \quad 0.1 \pm 0.2$

$0.2 \pm 0.5 \quad 0.2 \pm 0.3$

$0.3 \pm 0.4 \quad 0.4 \pm 0.7$

$0.4 \pm 1.0 \quad 0.2 \pm 0.4$

$0.3 \pm 0.4 \quad 0.2 \pm 0.4$

$0.3 \pm 0.4 \quad 0.3 \pm 0.4$

$0.3 \pm 0.5 \quad 0.3 \pm 0.7$

$0.2 \pm 0.3 \quad 0.4 \pm 0.6$

$0.3 \pm 0.4 \quad 0.4 \pm 0.5$

$0.3 \pm 0.4 \quad 0.4 \pm 0.6$

$0.1 \pm 0.2$

$1.5 \pm 1.1$

$0.5 \pm 0.6$

$0.5 \pm 0.5$

$0.4 \pm 0.4$

$0.4 \pm 0.4$

$0.5 \pm 0.4$

$0.4 \pm 0.4$

$0.3 \pm 0.3$

$0.4 \pm 0.4$

$0.1 \pm 0.2$

$1.8 \pm 1.9$

$0.9 \pm 0.8$

$0.8 \pm 0.8$

$0.7 \pm 0.9$

$0.7 \pm 0.9$

$0.9 \pm 1.1$

$0.6 \pm 0.6$

$0.6 \pm 0.8$

$0.6 \pm 0.8$

$33.7 \pm 8.1$

$1.0 \pm 0.0$

$--$

$8.5 \pm 1.2$

$3.6 \pm 2.6$

$5.1 \pm 1.8$

$5.6 \pm 2.3$

$5.9 \pm 1.9$

$6.9 \pm 2.1$

$7.6 \pm 1.4$

$6.9 \pm 2.2$

$6.7 \pm 2.2$
$37.3 \pm 12.7$

$1.0 \pm 0.2$

--

$8.7 \pm 1.1$

$3.7 \pm 2.5$

$6.5 \pm 2.1$

$6.3 \pm 2.2$

$5.0 \pm 1.7$

$8.0 \pm 1.8$

$8.1 \pm 1.6$

$7.8 \pm 1.9$

$7.8 \pm 2.2$ 


\begin{tabular}{|c|c|c|c|}
\hline $\begin{array}{l}\text { Trustworthiness experimenter B a } \\
\text { (who gave verbal suggestions) }\end{array}$ & $5.6 \pm 3.0$ & $6.4 \pm 2.2$ & $7.8 \pm 1.9$ \\
\hline Competent ${ }^{a}$ & $7.6 \pm 2.0$ & $7.6 \pm 1.8$ & $7.8 \pm 2.0$ \\
\hline Sympathetic a & $6.9 \pm 2.4$ & $6.5 \pm 2.3$ & $7.0 \pm 2.5$ \\
\hline Honest $^{a}$ & $4.2 \pm 2.6$ & $5.4 \pm 2.3$ & $7.4 \pm 2.5$ \\
\hline Concerned what experimenter thought ${ }^{b}$ & $4.2 \pm 3.0$ & $3.0 \pm 2.2$ & $4.1 \pm 2.9$ \\
\hline Changed responses to help experimenter ${ }^{b}$ & $1.7 \pm 1.8$ & $2.0 \pm 2.1$ & $1.9 \pm 2.3$ \\
\hline Owed it to experimenter to report less or more pain ${ }^{c}$ & $4.2 \pm 1.7$ & $4.4 \pm 2.0$ & $5.2 \pm 0.9$ \\
\hline Provided answers experimenter wanted to hear ${ }^{b}$ & $1.9 \pm 2.0$ & $1.9 \pm 2.2$ & $1.5 \pm 1.9$ \\
\hline \multicolumn{4}{|l|}{ Psychological characteristics } \\
\hline LOT-R - optimism & $15.7 \pm 3.9$ & $16.1 \pm 3.5$ & $15.7 \pm 3.8$ \\
\hline GTS - general trust & $3.7 \pm 0.5$ & $3.4 \pm 0.6$ & $3.5 \pm 0.6$ \\
\hline PCS - pain catastrophizing & $19.0 \pm 9.5$ & $14.2 \pm 8.3$ & $16.1 \pm 7.5$ \\
\hline SMS-R ability to modify self-presentation & $23.3 \pm 4.2$ & $21.7 \pm 5.0$ & $22.8 \pm 4.6$ \\
\hline SMS-R sensitivity to others & $20.4 \pm 5.0$ & $19.9 \pm 3.1$ & $20.6 \pm 3.5$ \\
\hline STAI-T - trait anxiety & $40.7 \pm 10.1$ & $39.5 \pm 8.6$ & $41.4 \pm 8.7$ \\
\hline STAI-Ss - state anxiety (baseline) & $34.1 \pm 11.7$ & $34.0 \pm 9.0$ & $33.4 \pm 8.7$ \\
\hline
\end{tabular}




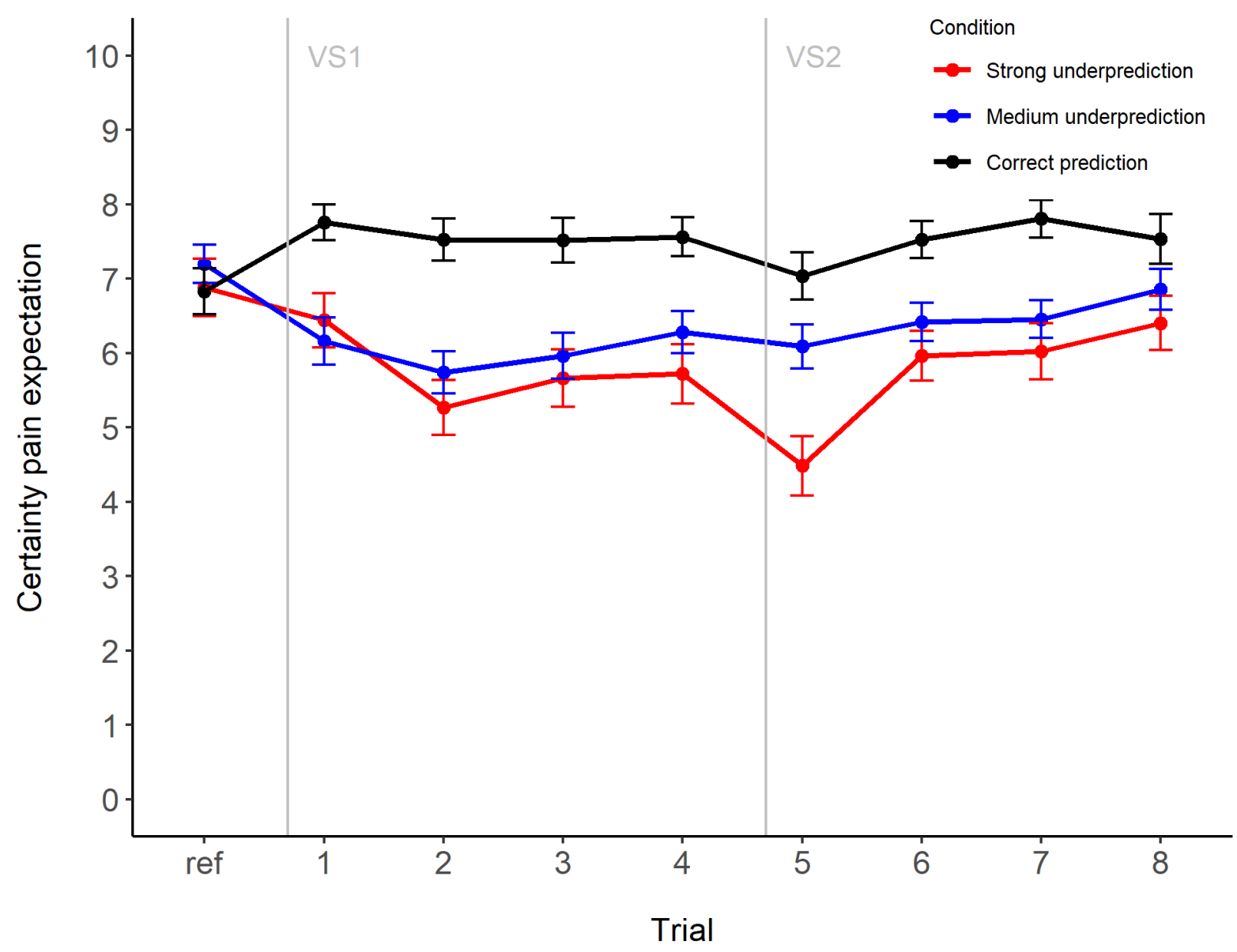

Figure SDC-9.

Certainty of pain expectation ratings (mean \pm standard error) for the reference stimulus and the 8 test stimuli per condition in Study 2.

ref $=$ reference stimulus; VS = verbal suggestion . 


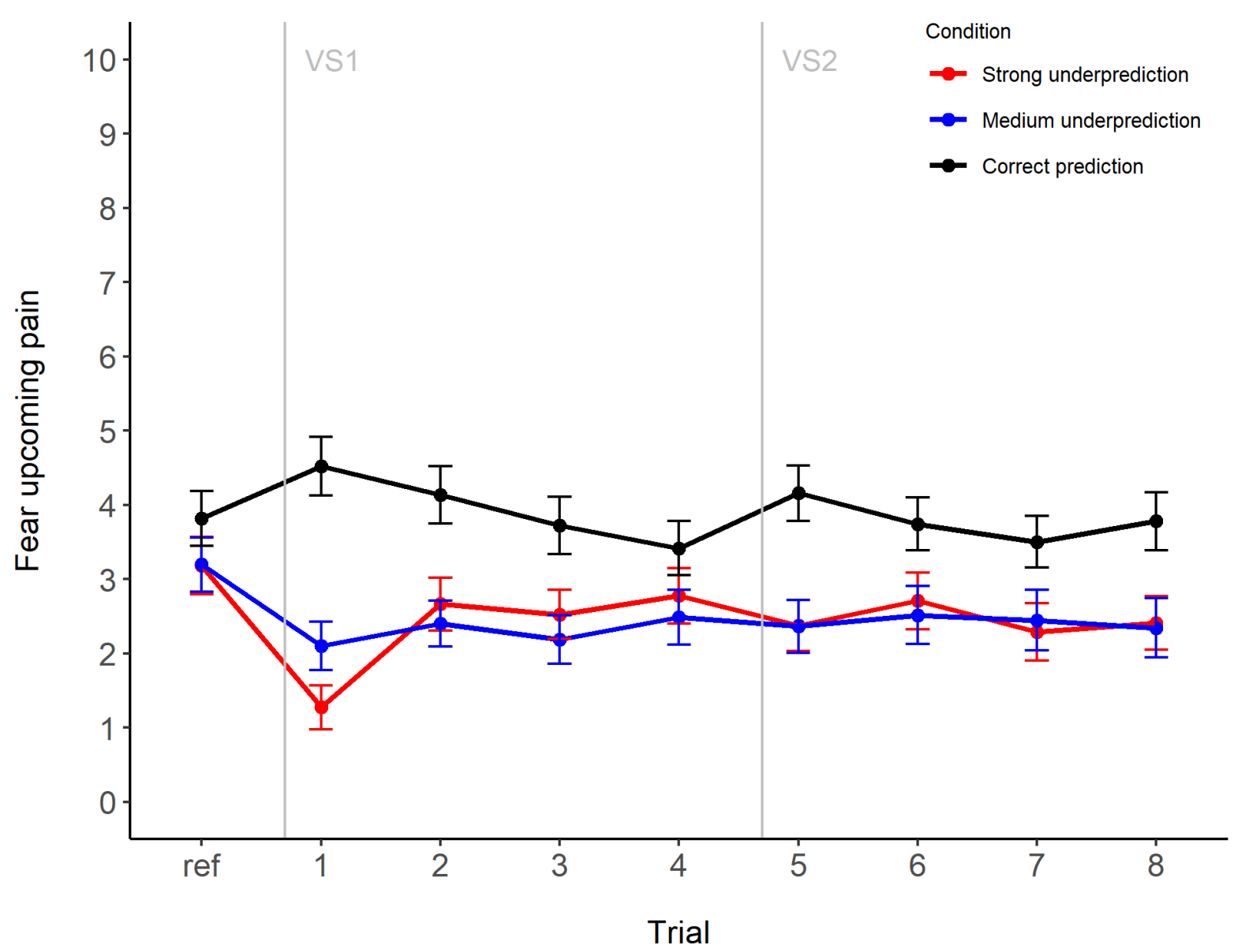

Figure SDC-10.

Fear of upcoming pain ratings (mean \pm standard error) for the reference stimulus and the 8 test stimuli per condition in Study 2.

ref $=$ reference stimulus; $V S=$ verbal suggestion 


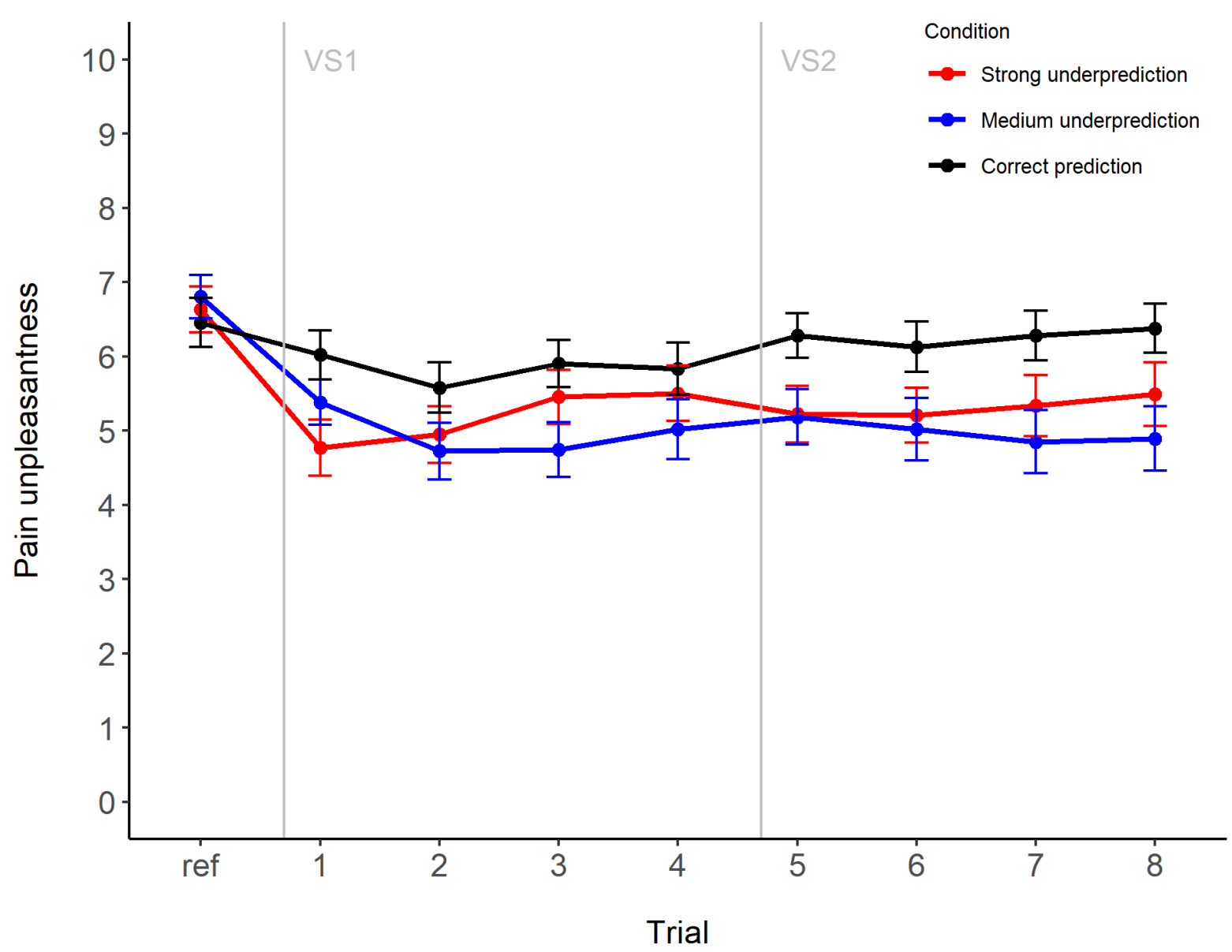

Figure SDC-11.

Pain unpleasantness ratings (mean \pm standard error) for the reference stimulus and the 8 test stimuli per condition in Study 2.

ref $=$ reference stimulus; $V S=$ verbal suggestion 


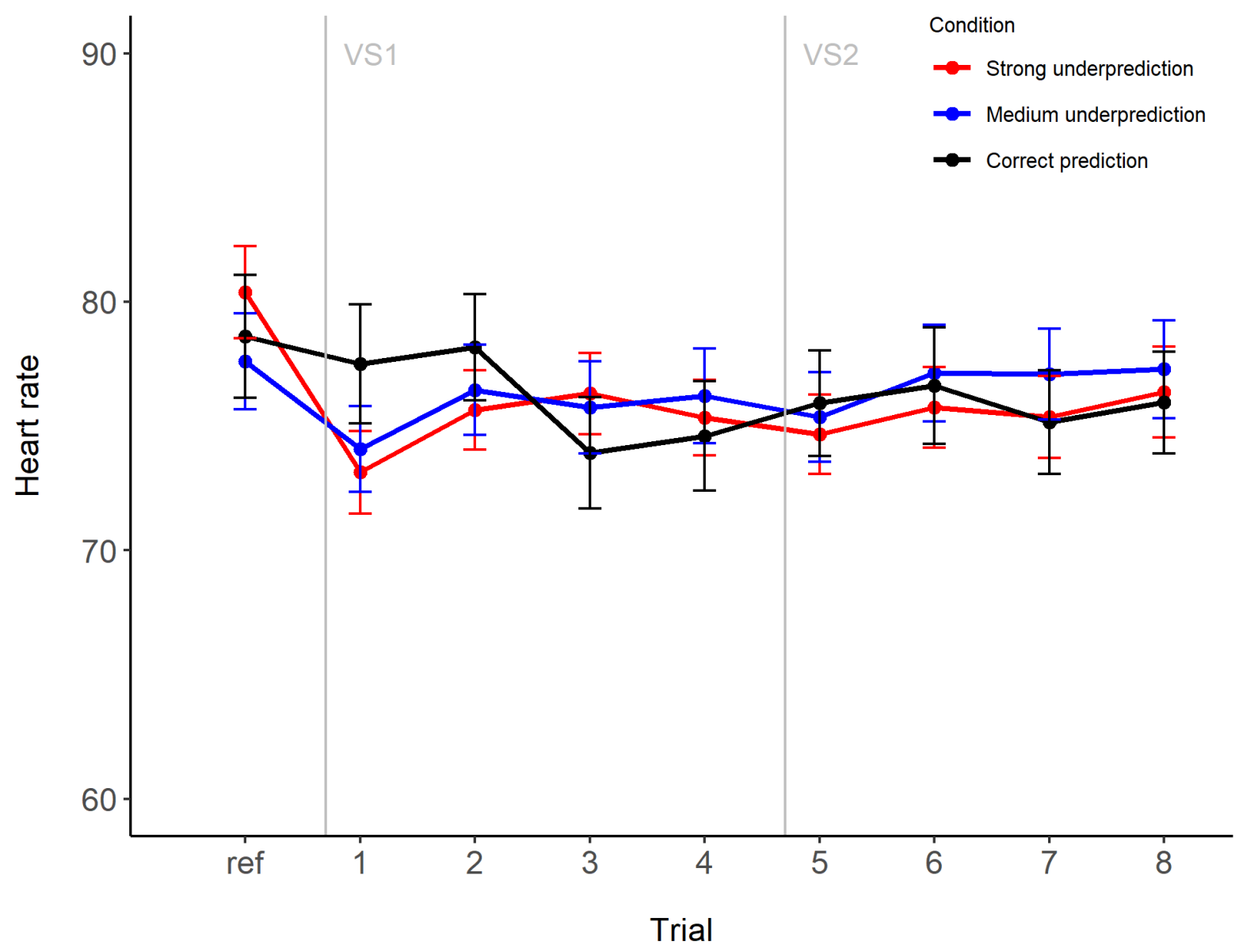

Figure SDC-12.

Heart rate (mean \pm standard error) for the reference stimulus and the 8 test stimuli per condition in Study 2.

ref $=$ reference stimulus; $V S=$ verbal suggestion . 


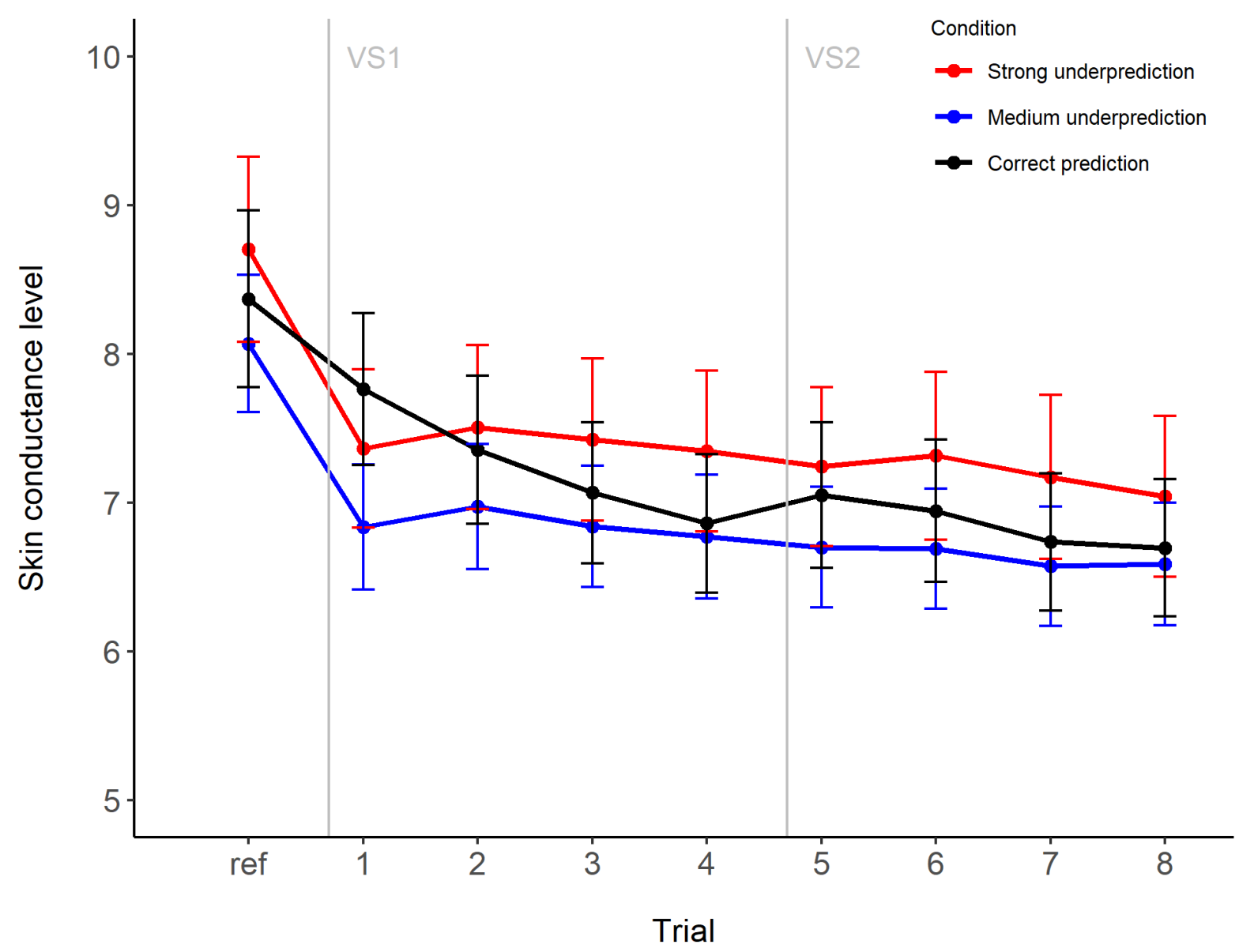

Figure SDC-13.

Skin conductance level (mean \pm standard error) for the reference stimulus and the 8 test stimuli per condition in Study 2.

ref $=$ reference stimulus; VS = verbal suggestion. 


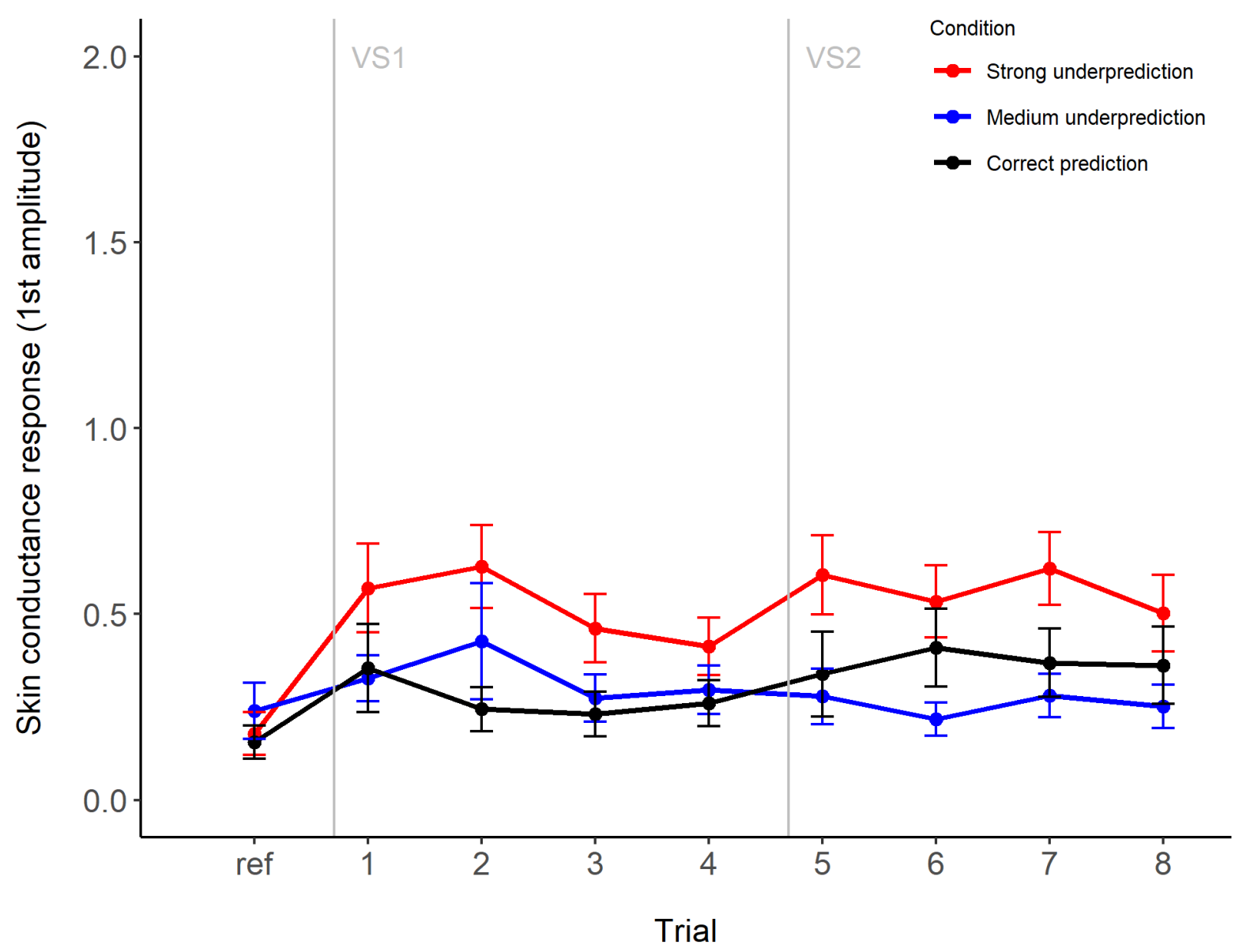

Figure SDC-14.

Amplitude first skin conductance response (mean \pm standard error) for the reference stimulus and the 8 test stimuli per condition in Study 2.

ref $=$ reference stimulus; VS = verbal suggestion . 


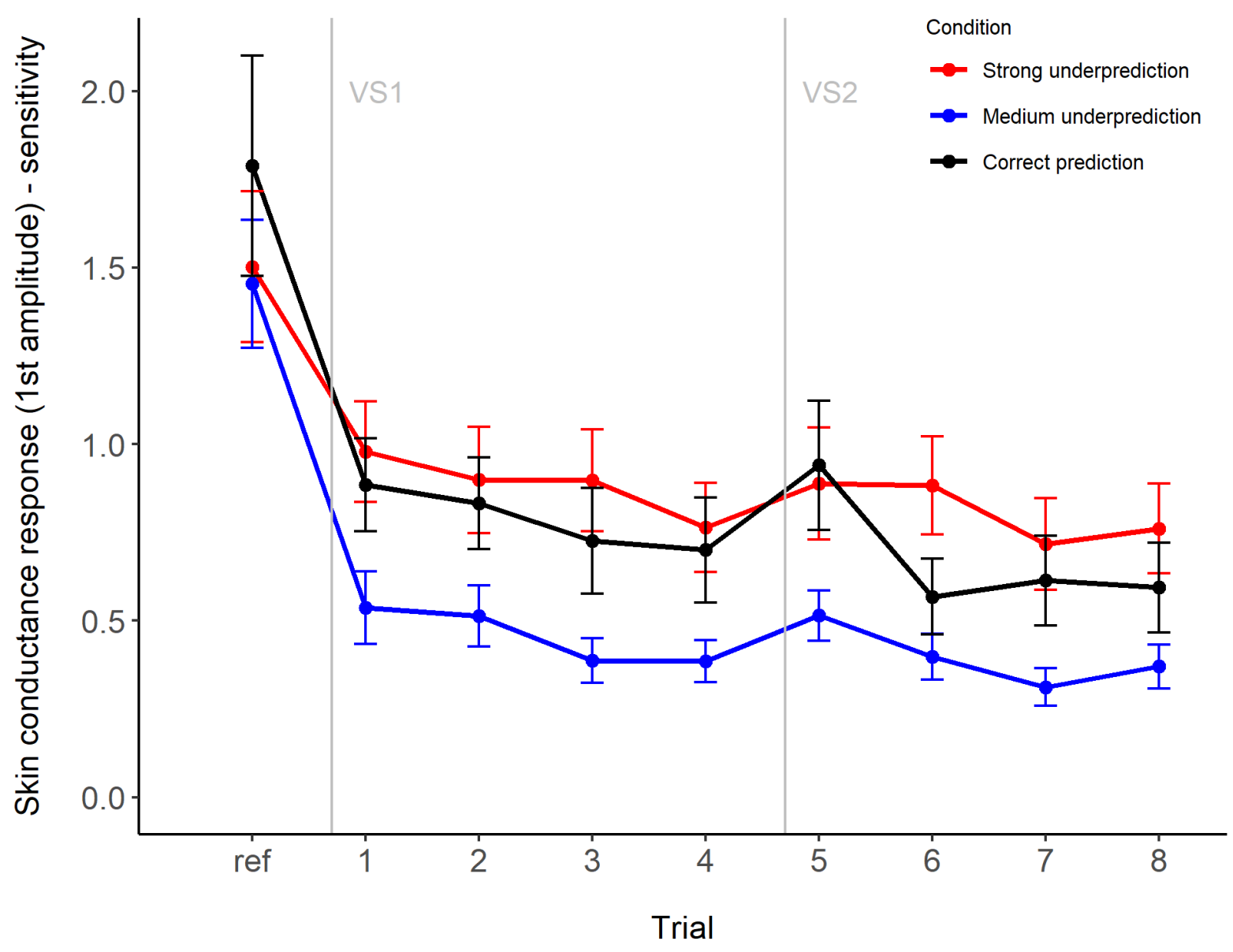

Figure SDC-15.

Amplitude first skin conductance response, with extended latency window (mean \pm standard error) for the reference stimulus and the 8 test stimuli per condition in Study 2.

ref $=$ reference stimulus; $V S=$ verbal suggestion. 